\title{
Nonequilibrium theory of scanning tunneling spectroscopy via adsorbate resonances: nonmagnetic and Kondo impurities
}

\author{
M. Plihal and J. W. Gadzuk \\ National Institute of Standards and Technology, Gaithersburg, MD 20899
}

We report on a fully nonequilibrium theory of the scanning tunneling microscopy (STM) through resonances induced by impurity atoms adsorbed on metal surfaces. The theory takes into account the effect of the tunneling current and finite bias on the system and is valid for arbitrary intra-adsorbate electron correlation strength. It is thus applicable to the recent STM experiments on Kondo impurities. We discuss the finite temperature effects and the consequences of atomic scale resolution of the STM for the spectral property of such systems. We find that the tip position affects the resonance lineshapes in two ways. As a function of distance from surface, the lineshapes vary due to the different extent of the adsorbate and metal wavefunctions into the vacuum. However, we do not expect large variations in lineshapes unless the tunneling into the tightly bound adsorbate states is considerable or when nonequilibrium effects are significant. As a function of lateral tip position, lineshapes should not change significantly on length scales of $R_{\|} \leq 10 \AA$ under typical experimental conditions when the electrons tunnel into the perturbed bulk conduction states hybridized with the outer shell $s p$ adsorbate orbitals. Tunneling into surface states on (111) surfaces of noble metals should be important for observation of the resonance at larger distances $(>10 \AA)$ and oscillatory variations in the lineshape should develop. This long range behavior has not been resolved in the recent experiments with Kondo impurities. The temperature dependence of the Kondo resonance cannot be deduced directly from the differential conductance as the thermal broadening of the tip Fermi surface produces qualitatively similar effects of comparable and larger magnitude. Careful deconvolution is necessary to extract the temperate dependence of the Kondo resonance. The finite-bias current-induced nonequilibrium effects in tunneling through Kondo impurities should produce characteristic broadening of the resonance in the case of strong hybridization of the discrete state with the STM tip.

PACS numbers:61.16.Ch,72.10.Fk

\section{INTRODUCTION}

A considerable body of experience and wisdom within the area of solid state tunneling phenomenon was built up throughout tunneling's "Golden Era of the Sixties". It was during this period that many of the defining fundamental ideas, basic theoretical strategies and methodologies, and broad scope of new applications for tunnel structures were first realized. A general introduction to many of these achievements can be found in a number of comprehensive volumes ${ }^{1-3}$ and in the Nobel Lectures of Esaki, Giaver, and Josephson, who were awarded the 1973 Nobel Prize in Physics for "their [independent] discoveries regarding tunneling phenomena in solids" ${ }^{4}$. It is against this background that the astounding achievements in contemporary tunneling studies utilizing the single atom spatial resolution of the scanning tunneling microscope (STM) are most meaningfully considered $^{5-10}$. One phenomenon of key interest here which was first considered in the "Golden Era" is that of impurity/adsorbate-assisted elastic tunneling. Two bodies of work are particularly relevant to the present study. The first is the recognition by Appelbaum and coworkers of the possible role of the Kondo effect ${ }^{11-13}$ in determining certain current-voltage characteristics (e.g. "zero-bias anomalies") of metal-oxide-metal tunnel junctions containing localized paramagnetic impurity states near the metal-oxide interfaces ${ }^{14}$. Second are the resonance tunneling studies involving valence electronic levels of single atoms adsorbed on metal surfaces, as probed in a field emission microscope configured for energy analysis (thus enabling electron spectroscopy) of the field emitted electrons ${ }^{15-17}$. Many years later, useful parallels between the theory of single atom resonance tunneling developed in the "Golden Era" and the theory of the STM, in the single-atom-tip limit, were unambiguously established ${ }^{18}$. Further discussion of these issues from the past will be offered throughout the text, when appropriate.

The basis for continuing interest and excitement in impurity/adsorbate- assisted tunneling is that the transparency of tunnel junctions can be dramatically enhanced by the presence of states localized within the barrier when they are in resonance with the tunneling electrons. Tunneling through such states is, for example, the origin of conductance fluctuations quantum dots exhibit in the Coulomb blockade ${ }^{19}$. The tunneling probability in the presence of a "barrier" state is proportional to the spectral density produced by the hybridization of the localized state with the conduction electrons and in many situations the current is given by the Breit-Wigner formula

$$
I \propto \frac{\Gamma^{2}}{\left(\omega-\epsilon_{0}\right)^{2}+(\Gamma / 2)^{2}} .
$$

Here $\Gamma$ is the width at half maximum of the resonance produced by the hybridization with the conduction electrons in the right and left lead and $\epsilon_{0}$ is the energy of the local state. The value of $\Gamma$ depends on the height and width of the barrier potential between the central region and the leads. 
Recently, enhancements in the zero bias conductance in quantum dots due to the Kondo effect have been observed $^{20}$. It had been shown earlier that $(d I / d V)$, the zero-bias differential conductance, is proportional to the Fermi level density of states of the Kondo resonance on the quantum dot. Similarly, the Kondo resonance has been spectroscopically observed on single magnetic impurities adsorbed on metal surfaces using the $\mathrm{STM}^{21,22}$. However in the case of the spectroscopic STM experiments, the resonance at the Fermi level appears to have an asymmetric shape and cannot be interpreted simply in terms of the local density of states of the impurity atom. Rather, the electron tunneling current - being a coherent quantum effect - is a result of interference between competing tunneling channels, as will soon be detailed. Unlike in the quantum dot where the tunneling can take place with appreciable magnitude only through the quantum dot region, the apparent tunneling current from the STM tip to the surface can either go through the resonance localized on the impurity or directly into the conduction states of the surface. The distinction between the conduction and local states will be discussed later. The notion that the tunneling conductance is proportional to the local density of states near the STM tip must then be modified.

In addition to its most common use for observing/determining atomic geometrical structure at surfaces, the STM is used as a sensitive probe of surface electronic structure. Various theoretical approaches to the STM conductance employ the tunneling Hamiltonian introduced by Bardeen ${ }^{23}$ and Golden Rule type expressions in which under certain limiting conditions of practical interest the STM conductance is indeed determined by the surface density of electronic states near the STM tip $^{24,25}$. Tersoff and Hamann ${ }^{24}$ developed a widely used model of the scanning tunneling microscope that includes the three dimensionality and spatial resolution of the tip.

The generic problem of a discrete state interacting with a continuum of states arises in many different areas of physics and chemistry ${ }^{26}$. In condensed matter physics a frequently occurring realization is the electronic state of an impurity atom immersed within a host lattice. ${ }^{27,28}$ In the case of magnetic impurities, the interaction gives rise to nontrivial phenomena such as the Kondo effect ${ }^{11-13}$.

Within the context of atomic physics, Fano discussed related effects, as they might appear on observable absorption lineshapes or resonant electron scattering cross sections which are due to the configuration interaction (CI) that couples a discrete two-electron excited atomic state with a continuum of ionization states ${ }^{29,30}$. Whilst the "natural" lineshape of the resonance is Lorentzian, when studied by experiments in which an external probe interacts with the system, the resonance can appear to have an asymmetric lineshape. Such lineshapes are referred to as Fano resonances. Fano found that an asymmetry in absorption lineshapes is due to interference between the excitation or decay into CI-mixed discrete and continuum states which both couple to the external probe. If the coupling between the probe and continuum is expressed in terms of an energy independent matrix element $t_{c}$ and the interaction between the probe and the localized state (which has already been diluted by admixture into the continuum) by the matrix element $\tilde{t}_{a}$, then the lineshape detected has the form

$$
I \propto \frac{\left(q+2\left(\omega-\epsilon_{0}\right) / \Gamma\right)^{2}}{1+\left(2\left(\omega-\epsilon_{0}\right) / \Gamma\right)^{2}},
$$

where $q \equiv \tilde{t}_{a} /\left(2 \pi V t_{c}\right)$ with $V$ being the hybridization (or CI) matrix element between the local state and continuum. The latter coupling results in the discrete state acquiring a width $\Gamma=2 \pi \rho_{s} V^{2}$ ( $\rho_{s}$ is the density of continuum states).

In the present paper, we consider the problem of the discrete state embedded in a continuum using a probe such as the STM that has atomic scale spatial resolution. This work has been motivated by the recent STM experiments involving single Kondo impurities ${ }^{21,22}$. The resonance observed in the conductance was interpreted by the authors in terms of the Fano interference. The fit of the resonance to the Fano formula ${ }^{21}$ - generalized to the case where intra-atomic Coulomb interaction on the impurity is taken into account - was based on the assumption that the tunneling into an Anderson impurity can be extended to include the tunneling into the continuum in a straightforward way. Upon further consideration, it appears that this generalization of the Fano result to the case of STM conductance is not as straightforward as has been presumed. In the present paper we obtain a more complicated expression than the elementary Fano formula, one which accounts for the correct asymptotic behavior for large tip-impurity separation. In particular, when the dependence of the probe's distance from the local state is properly included, then observable consequences of the local state admixture with the conduction electrons show the correct asymptotic long range behavior in the large tip-to-impurity-separation-limit as they obviously should.

The difference between the Fano lineshape and the result obtained here is due to the different nature of the probe in the "multi-center" STM configuration (one "center" on the impurity/discrete state, the other on the STM tip/probe) compared with the "single-site" atomic physics processes. While Fano was concerned with light absorption or electron scattering where the system under study was always at the probe's focus, the outcome of STM experiments must depend upon the variable spatial position of the probe (tip) with respect to the discrete state under investigation. Put another way, for the atomic physics applications considered by Fano, both the discrete state coupled to the continuum and also the initially excited decaying or autoionizing state (the "probe" state in our STM language) are atomic states spatially localized at the same site by the same atomic central potential. In contrast, the "S" (=scanning!) in STM assures that the tip, hence initial excited state, can be indepen- 
dently located with respect to the position of the "discrete state coupled to the continuum" and it is this extra degree of freedom that enriches the potential information content in STM lineshape analysis, but also requires a much more detailed theoretical treatment than merely fitting the atomic physics Fano lineshape, Eq. (1.2), to position-dependent STM spectra. This will be expanded upon in depth later. This realization demonstrates the importance and crucial need for considering the measurement process in quantum mechanical observations.

While the structuring of this comprehensive paper is based on a logical development of the subject matter, it may be useful to present a roadmap of key points and results to guide the casual as well as the dedicated reader. In section II, we introduce the model of the system and discuss our approximations of the tunneling matrix elements resulting in Eqs. (2.13), (2.14), and (2.17). In section III and the supporting appendices (A-C), we develop the general nonequilibrium theory of STM tunneling current and conductance in the presence of an adsorbate induced resonance. The more familiar equilibrium limit, asymptotically exact for large tip-surface separation but pragmatically useful even for moderate $\sim 5 \AA$ separations, is treated as a special case in III B. The relationship between the equilibrium tunneling resonance lineshape observable in STM experiments and the asymmetric Fano lineshape is established in III C (Eqs. 3.24 and 3.25). The nonequilibrium contribution to the lineshape treated as a correction to the equilibrium limit is taken up in III D. The differential conductance is introduced in IIIE. The crucial role of the substrate electronic structure is explicitly considered in section III F where the substrate Green's function is evaluated for a jellium surface and in III G where surface states and real electronic structure effects are discussed qualitatively.

In section IV, we illustrate the predictions and consequences of our theory on two models for the adsorbate: nonmagnetic and Kondo. Using a jellium substrate model, we first discuss the common features of the two adsorbate models in terms of the non-interacting Anderson model in IV A. Four different families of spectroscopic lineshape variations are taken up: dependence on (1) the relative strength of the tip-to-adsorbate vs. tipto-substrate tunneling and on the substrate electronic structure; (2) tip-surface separation; (3) lateral tip position; (4) temperature due to Fermi level smearing. We explain why no variations in the resonance lineshape should be observed for lateral tip positions on length scales $\sim(1-2) \AA$ - characteristic of the bulk $k_{F}-$ and only small lineshape variations should be expected for vertical tip variations (in experimentally accessible range). We leave the discussion of the tip and bias effects on lineshapes to section IV B 3.

Tunneling characteristics specific to Kondo systems are presented in IV B. We begin with conceptual issues in IV B 1. We then discuss the recent experiments on $C o / A u(111)^{21}$ and $C e / A g(111)^{22}$ and relate our work to related theoretical papers ${ }^{31,32}$ ( IV B 2). In particular, we show that the temperature dependence of the Kondo resonance is not easily extractable from the temperature dependence of the differential conductance. We reiterate that the stability of the experimental lineshape with the tip position is to be expected. Variations in the lineshapes would, however, occur at larger distance due to tunneling into the surface state. The effect of adsorbatetip hybridization and bias induced nonequilibrium on the current (conductance) vs. bias measurements in Kondo systems are dealt with in section IV B 3. Finally, an enumeration of specific conclusions is offered in section $\mathrm{V}$.

\section{MODEL AND APPROXIMATIONS}

Models of scanning tunneling microscopy are abundant in the literature of the last two decades and standard texts exist ${ }^{7-10}$. We approach the problem as a nonequilibrium process and discuss the corrections to the Tersoff-Hamann formulation ${ }^{24}$. Our intent is to develop such theory under general and self-consistent assumptions that accurately capture most of the qualitative aspects involved and do so in a way that make extension to more realistic calculations formally straightforward. We focus on the tunneling through adsorbate resonances. Throughout this paper, we adopt the convention that the energies are measured with respect to the respective Fermi levels of the substrate and tip unless specified otherwise and set $\hbar=1$. When the tip is biased we explicitly shift the tip energies.

\section{A. Model of the studied system}

We consider a system which consists of a clean metallic surface with a single impurity atom adsorbed on it. The STM will be used to study the system by means of tunneling through a resonance produced by an electronic state of the impurity, such as the $5 f$ orbital of $\mathrm{Ce} / \mathrm{Ag}(111)^{22}$ or $3 d$ orbital in $C o / A u(111)^{21}$. Unless otherwise noted, we place the origin of the coordinate system at a point on the surface of the metal directly below the adsorbate. This means that the position of the impurity is $\vec{R}_{0}=\left(0,0, Z_{0}\right)$. The system without the probe is described by the degenerate Anderson Hamiltonian

$$
\begin{aligned}
H_{s}\left(\vec{R}_{0}\right) & =\sum_{a} \epsilon_{0}\left(\vec{R}_{0}\right) c_{a}^{\dagger} c_{a}+\sum_{a>a^{\prime}} U\left(\vec{R}_{0}\right) n_{a} n_{a^{\prime}}+ \\
& +\sum_{k a} \epsilon_{k} c_{k a}^{\dagger} c_{k a}+\sum_{k a}\left\{V_{k a}\left(\vec{R}_{0}\right) c_{k a}^{\dagger} c_{a}+\text { H.c. }\right\} .
\end{aligned}
$$

Here, $\epsilon_{0}$ is the energy of the impurity state $\psi_{\sigma}(\vec{r})$, which we assume may be a multiplet of states described collectively by the quantum number $a \equiv(m \sigma)$. In the simplest case, $a$ correspond to the spin $\sigma$, but it may also include orbital degeneracy $(m)$ in more complicated cases. In this paper, we discuss at most spin degenerate states with 
$a=\sigma$ and $N=2$ (degeneracy). We denote by $c_{a}^{\dagger}$ the creation operator for this state. The $\epsilon_{k}$ is the conduction band state energy - independent of $\sigma$ in the absence of magnetic field - with $c_{k a}^{\dagger}$ being the creation operator for the corresponding Bloch state with symmetry (spin) $a$ common with the impurity state, and $V_{k a}$ is the matrix element for hybridization between the impurity and conduction states. The second term in (2.1) corresponds to the intra-atomic Coulomb interaction between electrons in the impurity state $\psi_{a}$.

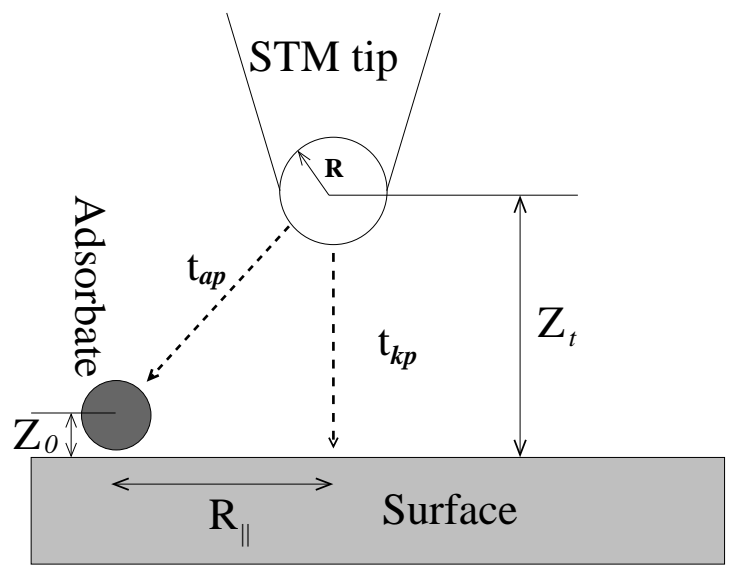

FIG. 1. schematic picture of the STM

If the renormalized energy $\epsilon_{0}$ lies within the conduction band, the bound state broadens into a resonance which in the wide-band limit and with $U=0$, has a Lorentzian shape

$$
\rho_{0}(\omega)=\frac{1}{2 \pi} \frac{\Gamma}{\left(\omega-\epsilon_{0}\right)^{2}+(\Gamma / 2)^{2}}
$$

where

$$
\Gamma=2 \pi \rho_{s} V^{2}
$$

with $\rho_{s}$ and $V$ the assumed-energy-independent density of conduction states and hybridization matrix element from (2.1). In most of our later considerations however, we will retain an energy dependence in the model density of states, $\rho_{s}(\omega)$.

\section{B. Interaction of the system with the STM tip}

When the STM tip is brought near the impurity, electrons can tunnel between the tip and the adsorbate state. This situation is expressed by adding an interaction term to the Hamiltonian

$$
H_{a t}\left(\vec{R}_{t}, \vec{R}_{0}\right)=\sum_{p a}\left\{t_{a p}\left(\vec{R}_{t}, \vec{R}_{0}\right) c_{a}^{\dagger} c_{p}+H . c .\right\} .
$$

The tip states are denoted by subscript $p$ while the conduction states of the metal by subscript $k$. The transfer matrix $t_{a p}$ depends on the position of both the adsorbate $Z_{0}$ and the tip $\vec{R}_{t} \equiv\left(\vec{R}_{\|}, Z_{t}\right)$.

If the STM only coupled to the discrete state with transfer amplitude $t_{a}$ then the conductance would, in the wide-band limit $\left(t_{a p} \equiv t_{a}\right.$, independent of $\left.p\right)$, be determined by

$$
G \propto\left|t_{a}\right|^{2} \frac{\Gamma}{\left(\omega-\epsilon_{0}\right)^{2}+(\Gamma / 2)^{2}}
$$

and the conductance would thus be directly related to the impurity density of states. This is reminiscent of the defining characteristics from field emission resonance tunneling spectroscopy in which tunneling from the substrate to vacuum is disproportionately smaller than that from "good" adsorbates ${ }^{15-17,33}$. However, since in the STM geometry, tunneling directly between the tip and the metal surface can be comparable to (or in excess of) that between the tip and the impurity, the conductance exhibits a more complex behavior than that of a simple impurity local density of states. We take such processes into account through

$$
H_{s t}\left(\vec{R}_{t}\right)=\sum_{p k}\left\{t_{k p}\left(\vec{R}_{t}\right) c_{k}^{\dagger} c_{p}+H . c .\right\}
$$

where the tunneling matrix element $t_{k p}$ depends on the position of the tip $\vec{R}_{t}$. In the rest of this section, we will address the issue of the probe's effect on the system itself. This is particularly important when the tip is brought very close to the adsorbate so that the tunneling $t_{a p}$ and $t_{k p}$ are comparable with $V_{k a}$. In this case, the width $\Gamma$ of the adsorbate resonance is no longer given by (2.3) but rather by

$$
\Gamma=2 \pi\left(\rho_{s} V^{2}+\rho_{t} t_{a}^{2}\right)
$$

The effect of the tip on the conduction states can also be important. However, the most important consequence of the tip perturbation comes when finite bias is applied across the tunnel junction. The system will be out of equilibrium and the problem of the system-probe must be approached self-consistently with the tip included in the system it is probing. The typical operational mode of the STM during imaging and spectroscopic measurements is such that the tip distance from the adsorbate is several atomic units larger than the adsorbate-surface separation and therefore $\left|t_{a p}\right|,\left|t_{k p}\right| \ll\left|V_{k a}\right|$. In this limiting case, the probe has no effect on the adsorbate-metal complex other than as a source of hot tunneling electrons. This is a reasonable assumption as long as the tip separation is large enough to justify the approximation $\left|t_{a p}\right|,\left|t_{k p}\right| \ll$ $\left|V_{k a}\right|$. 


\section{Model of the STM tip}

An important property of the tip is its spatial resolution, as discussed in great detail by many $6,18,24,34-36$. We will consider the tip to be well defined and terminated by a single atom through which the tunneling predominantly takes place. This is the s-wave model of Tersoff and Hamann ${ }^{24}$, see Fig 1. The important features are the following: (a) the tip Hamiltonian is

$$
H_{t}=\sum_{p} \epsilon_{p} c_{p}^{\dagger} c_{p}
$$

where $c_{p}^{\dagger}$ creates an electron in the state $\psi_{p}(\vec{r})$ with energy $\epsilon_{p}$ measured from the Fermi level of the tip $\epsilon_{F t}$; (b) when the tip is positioned near the surface, tunneling into and out of a state $\psi_{p}$ can take place; (c) the states are filled up to the chemical potential $\epsilon_{F t}$ controlled by the bias; (d) the tip states are characterized by a density of states which we denote by $\rho_{t}(\omega)=\sum_{p} \delta\left(\omega-\epsilon_{p}\right)$; and (e) the asymptotic form of the tip eigenstate $\psi_{p}$ in the vacuum region extending towards the metal surface is characterized by the atomic orbital of the apex atom. The wavefunction $\psi_{p}$ can be found based on simple physical arguments without solving the complete problem. If $\phi_{t}$ is the work function of the tip and $\kappa_{t} \equiv \sqrt{2 m_{t}^{*}\left(\phi_{t}-\epsilon_{p}\right)}$, then following Tersoff and Hamann,

$$
\psi_{p}(\vec{r}) \propto R e^{\kappa_{t} R} \frac{\exp \left(-\kappa_{t} r\right)}{r}
$$

where $\mathrm{R}$ is the radius of curvature of the tip about its center which is located at the origin of this "tip-defining" coordinate system. While (2.9) represents an "s-wave tip", more generally $\psi_{p}$ would carry whatever symmetry was possessed by the relevant atomic orbital centered at the tip apex ${ }^{10,18,34}$. The wavefunction tail, controlled by $\kappa$, depends on the bias and tip-surface separation. Both factors modify the height of the vacuum barrier, hence effective work function $\phi$ determining $\kappa$. These modification can be essentially included by renormalizing the wavefunction tails and the densities of states by position and energy dependent factors via the tunneling matrix elements. We discuss the tunneling matrix elements next.

\section{Approximations for the tunneling matrix elements}

An important role in our formulation is played by the tunneling (hybridization) matrix elements $V_{k a}, t_{a p}$, and $t_{k p}$ since they include the dependence on electronic structure and the tip and adsorbate position. The desired quantitative accuracy of the model for the tunneling process is to a large degree determined by the approximations made in the evaluation of these matrix elements. ${ }^{18,34,37}$ We do this first for a general adsorbatemetal system and then for a jellium model. It is rather straightforward to include band structure effects using a realistic electronic structure calculation of the substrate Green's function.

We begin with the discussion of the matrix elements $V_{k a}$ and $t_{k p}$ that contain the metal wavefunctions $\psi_{\vec{k}}(\vec{r})$. They have the form

$$
M_{k l}\left(\vec{R}_{l}\right)=\int d^{3} r \psi_{\vec{k}}^{*}(\vec{r}) v_{s l}(\vec{r} ; \vec{R}) \psi_{l}^{\prime}(\vec{r}),
$$

where $v_{s l}$ is the potential representing the mutual interaction of the two systems. The wavefunction $\psi_{\vec{k}}(\vec{r})$ is a Bloch state of the unperturbed metal and $\psi_{l}^{\prime}(\vec{r})$ is the wavefunction (in the coordinate system of the metal) of the adsorbate state $a$ in the case of $V_{k a}$ or the tip wavefunction in the case of $t_{k p}$. Either way, $\psi_{l}^{\prime}(\vec{r})$ can be written in terms of the wavefunction with the origin at the adsorbate (tip) as $\psi_{l}^{\prime}(\vec{r})=\psi_{l}\left(\vec{r}-\vec{R}_{l}\right)$, where $\vec{R}_{l}$ is the position of the adsorbate $\left(\vec{R}_{0}\right)$ or the tip apex atom $\left(\vec{R}_{t}\right)$ measured from a reference point on the surface. The energies $\epsilon_{k}$ of the metal electrons are written in terms of the perpendicular and parallel components as $\epsilon_{k} \equiv \epsilon_{k_{z}}+\epsilon_{k_{\|}}$. We follow the convention that $\epsilon_{k_{\|}}$is measured from the bottom of the 2-D band and $\epsilon_{k_{z}}$ is measured with respect to $\epsilon_{F s}$. For example, we write for the jellium model $\epsilon_{k z}=k_{z}^{2} / 2 m_{s}^{*}-D$ and $\epsilon_{k_{\|}}=k_{\|}^{2} / 2 m_{s}^{*}$, where $(-D)$ is the energy of the bottom of the band with respect to the Fermi level. The Bloch states can generally be written in the relevant region outside the metal as

$$
\psi_{n \vec{k}}(\vec{r})=e^{-\kappa_{n s} z} u_{n \vec{k}_{\|}}(\vec{\rho}, z) e^{i \vec{k}_{\|} \cdot \vec{\rho}}
$$

where $n$ is the band index, $u_{n \vec{k}_{\|}}(\vec{\rho}, z)$ is a function weakly dependent on $z$ outside the surface and periodic in $\vec{\rho}$, the electron coordinate in the plane of the surface. This form is equally valid for the metal band gap surface states that seem ubiquitous to STM studies on (111) noble metal surfaces since both the z-propagating Bloch states and the localized surface states are eigenstates of the same Hamiltonian with different eigenvalues at a given $k$. At the surface, evanescent states into the bulk that appear upon analytic continuation of the band structure into the domain of complex k-vectors cannot be rejected on the basis of physical considerations as they were for the perfectly periodic interior of the solid.

For jellium, $u_{n \vec{k}_{\|}}(\vec{r})$ is constant and the the metal states are then simply plane waves along the surface with exponentially decaying amplitude into the vacuum. Here $\kappa_{n s}=\sqrt{2 m_{s}^{*}\left(\phi_{s}-\epsilon_{n k_{z}}\right)}=\sqrt{2 m_{s}^{*}\left(\phi_{s}-\epsilon_{n k}+\epsilon_{n k_{\|}}\right)}$with $m^{*}$ the metal electron effective mass (number) and $\phi_{s}$ the height of the tunneling barrier for a Fermi level electron. For bias voltages much smaller than the work function, $\phi_{s}$ is equal to the metal work function. We omit the band index $n$ in the rest of the paper unless we explicitly discuss the electronic structure effects. We also define $\lambda_{\omega}^{-1}=\sqrt{2 m_{s}^{*}\left(\phi_{s}-\omega\right)}$, where the energy factor $\left(\phi_{s}-\omega\right)$ represents the effective tunneling potential barrier for an 
electron with energy $\omega$. For small bias voltages, $\omega \approx 0$, and we can replace $\lambda_{\omega}$, which depends weakly on energy in this range, by its Fermi level value $(\equiv \lambda)$.

We shift the integration variable in the integral (2.10) to an origin centered on the adatom (tip), which gives

$$
\begin{aligned}
M_{k l}\left(\vec{R}_{l}\right) & =\psi_{k}^{*}\left(\vec{R}_{l}\right) \int_{z>-Z_{l}} d^{3} r e^{-\kappa_{s} z} e^{-i \vec{k}_{\|} \cdot \vec{\rho}} \times \\
& \times \frac{u_{\vec{k}}^{*}\left(\vec{r}+\vec{R}_{l}\right)}{u_{\vec{k}}^{*}\left(\vec{R}_{l}\right)} v_{s a}^{\prime}\left(\vec{r}, R_{l}\right) \psi_{l}(\vec{r}) .
\end{aligned}
$$

Matrix elements of this type have been the focus of intense study in the context of charge transfer processes at surfaces. ${ }^{37-42}$ The main contribution to the integral in (2.12) comes from the region just outside the surface where the $z$-dependence of the metal states is that of a decaying exponential. In the case of both the adatom and the tip, the integrand is assumed to be well localized to the $z>-Z_{0}$ region by virtue of the spatial properties of $\psi_{l}$. Consequently, the integral will be reasonably constant for all $k_{\|} \leq 1 / r_{l}$ where $r_{l}$, the radial length scale for the atom or tip, sets the range of $k_{\|}$. Since, as will soon be demonstrated, other factors in the full problem will provide a much more severe $k_{\|}$cutoff, it is sufficient to represent the integral by a constant. We then write the matrix element approximately $M_{k l}\left(\vec{R}_{l}\right) \simeq M_{0} \psi_{\vec{k}}^{*}\left(\vec{R}_{l}\right)$, where $M_{0}$ is the overlap integral defined in (2.12) that contains the dependence of the matrix element on the symmetry of the atomic orbitals near the tip and on selection rules.

Since the distance of the adsorbate from the surface is small, it will be sufficient for the purpose of $V_{k a}$ to write the Bloch state in the form $\psi_{\vec{k}}\left(Z_{0}\right)=e^{-Z_{0} / \lambda} \psi_{\vec{k}}(0)$ with the decay constant independent of the k-vector. With the choice of our coordinate system $\vec{R}_{0}=\left(0, Z_{0}\right)$, we write $V_{k a}$ in the form of a separable product of a k-independent function of adsorbate position multiplied by a function of $\vec{k}$

$$
V_{k a}\left(\vec{R}_{0}\right) \simeq V_{a}\left(Z_{0}\right) \psi_{\vec{k}}^{*}(0)
$$

where $V_{a}\left(Z_{0}\right)=V_{0} e^{-Z_{0} / \lambda}$. We cannot make this last approximation in the tip-to-surface tunneling matrix element $t_{k p}$, since the interplay between $k$ and $Z_{t}$ dependence has an important role in the tunneling process. However, from the conceptual point of view, we find it convenient to isolate a $k$-independent dependence on the tip position in $t_{c}\left(Z_{t}\right)=t_{0} e^{-Z_{t} / \lambda}$ and write the matrix element in the form

$$
t_{k p}\left(\vec{R}_{t}\right)=t_{c}\left(Z_{t}\right) e^{Z_{t} / \lambda} \psi_{\vec{k}}^{*}\left(\vec{R}_{t}\right) .
$$

We note that the s-wave tip-to-surface tunneling matrix element $t_{k p}$ has been shown by Tersoff and Hamann ${ }^{24}$ under quite general assumptions to assume the form (2.14) with the tip wavefunction given by Eq. (2.9), which indicates that our simple qualitative arguments seem to be supported by more detailed analysis.
The tip-to-impurity matrix element

$$
\begin{aligned}
& t_{a p}\left(\vec{R}_{t}, \vec{R}_{0}\right)= \\
& =\int d^{3} r \psi_{\vec{p}}^{*}\left(\vec{r}-\vec{R}_{t}\right) v_{a t}\left(\vec{r} ; \vec{R}_{t}, \vec{R}_{0}\right) \psi_{a}\left(\vec{r}-\vec{R}_{0}\right) .
\end{aligned}
$$

depends on the position of both the adsorbate $\left(Z_{0}\right)$ and the tip $\left(\vec{R}_{t}\right)$. Since the tunneling from the tip takes place predominantly through the apex atom, the wavefunction $\psi_{p}$ in the last expression, a generalization of (2.9), can be written (with the tip at origin) as

$$
\psi_{\vec{p}}(\vec{r})=e^{\kappa_{t} R} \frac{e^{-\kappa_{t} r}}{r} \psi_{s}(\hat{r})
$$

where $\psi_{s}$ is the angular part of the orbital localized on the apex atom whose center is located at $\vec{R}_{t}$. The tunneling matrix element $t_{a p}$ will thus reflect the symmetry and spatial dependence of the states localized on the adsorbate and in the tip apex. Within the s-wave tip model, $\psi_{s}$ is just an innocuous constant. Since $\psi_{p}$ and $\psi_{a}$ appearing in Eq. (2.15) are both atomic-like functions in the relevant region of overlap centered respectively on the tip and on the adatom, in a broad sense $t_{a p}$ is similar to a common two-center hybridization/hopping integral defining the binding in a diatomic molecule ${ }^{43,44}$. Typically the magnitudes of these integrals are exponentially decreasing functions of their separation (possibly multiplied by a mildly oscillatory function accounting for the nodal structure of the atomic functions). Based on this analogy from quantum chemistry, $t_{a p}$ given by Eq. (2.15) should take the form

$$
t_{a p}\left(\vec{R}_{t}, Z_{0}\right) \approx t_{a} e^{-\left|\vec{R}_{t}-Z_{0} \hat{i}_{z}\right| / \alpha} \equiv t_{a}\left(\vec{R}_{t}, Z_{0}\right)
$$

Here, $\alpha^{-1} \approx\left(\kappa_{t}+\sqrt{2 m\left(\phi_{s}-\epsilon_{0}\right)}\right)$ is an effective decay constant evaluated for states at the Fermi level of the tip, $\left|\vec{R}_{t}-Z_{0} \hat{i}_{z}\right|=\sqrt{R_{\|}^{2}+\left(Z_{t}-Z_{0}\right)^{2}}$ is the tip-to-atom separation, as depicted in Fig. 1 , where $\vec{R}_{\|}$is the parallel component of $\vec{R}_{t}$. The decay constant $\kappa_{t}$ depends on the energy of the tip state $\left(\epsilon_{p}\right)$, but this dependence is very weak for small biases considered here and we neglect it. In this case, the matrix element is well approximated by the $p$ independent form $t_{a}\left(\vec{R}_{t}, Z_{0}\right)$. The matrix element $t_{a}$ may be taken real.

\section{THE NONEQUILIBRIUM THEORY OF THE TUNNELING CURRENT AND DIFFERENTIAL CONDUCTANCE}

The tunneling between the adsorbate-metal complex and a biased tip is a nonequilibrium process. Although we frequently make the assumption in this paper that the tip-system interaction is weak enough so that local equilibrium is maintained to a good approximation, the assumption is less valid when the tip is near the surface. 
For this reason, we develop our theory of the tunneling process within the Keldysh-Kadanoff ${ }^{45,46}$ framework for the nonequilibrium Green's functions and discuss the nonequilibrium corrections.

\section{A. General expression for the tunneling current in terms of nonequilibrium Green's functions}

We define the tunneling current as the flow of electrons through a closed surface around the tip. It is expressed in terms of the continuity equation as

$$
I=-e\left\langle\frac{d n_{t}(t)}{d t}\right\rangle
$$

where $n_{t}=\sum_{p} c_{p}^{\dagger} c_{p}$ is the number operator for the tip electrons, and the brackets signify the ensemble average, which in the local equilibrium case is the thermal average over the tip states. The time derivative is found from the Schrödinger equation of the total Hamiltonian of the tipsubstrate-adsorbate system $H_{t o t}=H_{s}+H_{t}+H_{a t}+H_{s t}$. Since the number operator commutes with $H_{s}$ and $H_{t}$, the only contribution comes from the interaction terms $H_{a t}$ and $H_{s t}$ and the current is

$$
I=\frac{2 e}{\hbar} \operatorname{Im}\left\{\sum_{k p} t_{k p}\left\langle c_{k}^{\dagger}(t) c_{p}(t)\right\rangle+\sum_{a p} t_{a p}\left\langle c_{a}^{\dagger}(t) c_{p}(t)\right\rangle\right\}
$$

where we omitted the arguments in $t_{k p}$ and $t_{a p}$ for convenience and used the relation $t_{a p}=t_{p a}^{*}$ and $t_{k p}=t_{p k}^{*}$. We write the arguments explicitly only when we wish to emphasize their dependence. We define the time loop Green's functions $G_{p a}\left(t, t^{\prime}\right)=-i\left\langle T_{C} c_{p}(t) c_{a}^{\dagger}\left(t^{\prime}\right)\right\rangle$ and $G_{p k}\left(t, t^{\prime}\right)=-i\left\langle T_{C} c_{p}(t) c_{k}^{\dagger}\left(t^{\prime}\right)\right\rangle$, where $T_{C}$ orders the times along a contour $C$ in the complex time plane. The contour can be taken to be the Kadanoff-Baym contour ${ }^{46}$, the Keldysh contour ${ }^{45}$, or a more general choice. The discussion of nonequilibrium Green's function is available in standard books and review articles ${ }^{46-48}$ and we refer the reader to these references for further details. Our notation follows closely that of the more detailed discussion in reference ${ }^{49}$. In the present paper, we are mainly interested in steady state tunneling current (time independent) and therefore we work with the Fourier transformed quantities in frequency rather than time space. The current can be written as

$$
I=\frac{2 e}{h} \operatorname{Im} \int_{-\infty}^{\infty} d \omega\left\{\sum_{k p} t_{k p} G_{p k}^{<}(\omega)+\sum_{a p} t_{a p} G_{p a}^{<}(\omega)\right\},
$$

where $G_{p k}^{<}(\omega)$ and $G_{p a}^{<}(\omega)$ are the Fourier transforms of $G_{p k}^{<}\left(t, t^{\prime}\right)=\left\langle c_{k}^{\dagger}\left(t^{\prime}\right) c_{p}(t)\right\rangle$ and $G_{p a}^{<}\left(t, t^{\prime}\right)=\left\langle c_{a}^{\dagger}\left(t^{\prime}\right) c_{p}(t)\right\rangle-$ the analytic pieces on the real time axis of the Green's functions introduced above. Equivalently, the current may be calculated from $\left\langle\frac{d n_{s}}{d t}+\frac{d n_{a}}{d t}\right\rangle$. It is easy to see that this approach also leads to the equation (3.2).

The problem of finding the current thus reduces to finding the "lesser" Green's functions $G_{p a}^{<}$and $G_{p k}^{<}$. This is done using the equation of motion method for the time ordered Green's functions in Appendix B and the rules for analytic continuation described in Appendix C. In order to see the interference between the two scattering channels giving rise to the Fano lineshape, it is also useful to express the current using (B2) and (B6) in Eq. (3.3) as

$$
\begin{aligned}
I & =\frac{2 e}{h} \operatorname{Im} \sum_{p} \int_{-\infty}^{\infty} d \omega \\
& \left\{G _ { p } ^ { ( 0 ) } \left[\sum_{a}\left|t_{p a}\right|^{2} G_{a}+\sum_{k a} t_{a p} t_{p k} G_{k a}+\right.\right. \\
& \left.\left.+\sum_{k a} t_{k p} t_{p a} G_{a k}+\sum_{k k^{\prime}} t_{p k^{\prime}} t_{k p} G_{k^{\prime} k}\right]\right\}
\end{aligned}
$$

The first term corresponds to the tunneling into the adsorbate state hybridized with the metal electrons. The fourth term is the contribution to the current from the direct tunneling into the conduction states perturbed by the presence of the discrete adsorbate state. The second and third terms give the interference between the two channels.

We substitute the solutions (B4) and (B9) for $G_{p k}$ and $G_{p a}$ from Appendix B into Eq. (3.3) and write the tunneling current in the form

$$
\begin{aligned}
I= & \frac{2 e}{h} \operatorname{Im} \int_{-\infty}^{\infty} d \omega \times \\
& \times \sum_{p p^{\prime}}\left\{G_{p p^{\prime}}^{R}(\omega) T_{p^{\prime} p}^{<}(\omega)+G_{p p^{\prime}}^{<}(\omega) T_{p^{\prime} p}^{A}(\omega)\right\},
\end{aligned}
$$

where the retarded function $T_{p p^{\prime}}^{R}\left(\vec{R}_{t}, \vec{R}_{0}, \omega\right)$ plays the role of the T-matrix for scattering of the tip electrons from the adsorbate-metal complex. It is defined by

$$
\begin{aligned}
T_{p p^{\prime}} & =\sum_{k} t_{p k} G_{k}^{0} t_{k p^{\prime}}+ \\
& +\sum_{a} \tilde{t}_{p a} G_{a}\left(t_{a p^{\prime}}+\sum_{k} \tilde{V}_{a k} G_{k}^{0} t_{k p^{\prime}}\right),
\end{aligned}
$$

with

$$
\tilde{V}_{k a}=V_{k a}+\sum_{p} t_{k p} G_{p}^{0} t_{p a}
$$

and

$$
\tilde{t}_{p a}=t_{p a}+\sum_{k} t_{p k} G_{k}^{0} V_{k a}
$$

the hybridization and tunneling matrix elements for the adsorbate modified by the tip-substrate interaction. The matrix $T_{p p^{\prime}}$ incorporates the properties of the tip as well as the adsorbate into the expression for current. We discuss its physical meaning more in the next section. 


\section{B. Equilibrium limit of the tunneling current at large tip-surface separation}

We define the equilibrium tunneling current as the large tip-surface separation limit of (3.5) when the tip, adsorbate, and substrate are all in local equilibrium. This is equivalent to keeping only the lowest order terms in $t_{k p}$ and $t_{p a}$. In our formalism, this is achieved by replacing $\tilde{G}_{p p^{\prime}} \rightarrow G_{p}^{0} \delta_{p p^{\prime}}$ in Eq. (3.5), $\tilde{V}_{k a} \rightarrow V_{k a}$ in $T_{p p^{\prime}}$, and by using the fluctuation-dissipation relation $G_{i}^{<}(\omega)=f_{i}(\omega) \rho_{i}(\omega)$. The subscript $i$ stands for tip (t), adsorbate (a), and metal (s), respectively. The adsorbate is in equilibrium with the metal, i.e. $f_{a}(\omega)=f_{s}(\omega)$. The matrix $T_{p p^{\prime}}$ is expressed entirely in terms of the Green's functions of the system and the tunneling matrix elements $t_{p k}, t_{p a}$. Since these matrix elements reflect the symmetry of the apex atom wavefunction, but are only weakly dependent on $p$ on the energy scale of the resonance width, the matrix $T_{p p^{\prime}}$ will also have this property. We therefore make an additional assumption that $\sum_{p} G_{p}^{0} T_{p p} \sim\left(\sum_{p} G_{p}^{0}\right) T_{t}$, where

$$
T_{t}=\sum_{k} t_{p k} G_{k}^{0} t_{k p}+\sum_{a} \tilde{t}_{p a} G_{a} \tilde{t}_{a p}
$$

is only a function of the atomic tip orbital independent of $p$. We define a tip-specific quantity observable by the STM, which is related to the local density of states

$$
\tilde{\rho}_{s a t}\left(\vec{R}_{t}, \vec{R}_{0} ; \omega\right)=-\frac{1}{\pi} \operatorname{Im} T_{t}^{R}\left(\vec{R}_{t}, \vec{R}_{0} ; \omega\right),
$$

and write the equilibrium current $I_{e q}\left(\vec{R}_{t}, \vec{R}_{0}, V\right)$ as

$$
I_{e q}=\frac{2 e}{h} \int_{-\infty}^{\infty} d \omega\left[f_{t}\left(\omega^{\prime}\right)-f_{s}(\omega)\right] \rho_{t}\left(\omega^{\prime}\right) \tilde{\rho}_{s a t}(\omega)
$$

where $\rho_{t}=\sum_{p} \delta\left(\omega-\epsilon_{p}\right)$ is the density of tip states and $\omega^{\prime}=\omega-e V$ with $V$ being the bias voltage. This equations is easily related to traditional formulations given in terms of an integral product of an electron "supply function" multiplied by a tunneling or transmission probability $^{1-3,15,17}$ when it is realized that $\tilde{\rho}_{\text {sat }}$, as defined here, already contains within it factors $\left(\propto|t|^{2}\right)$ representing the role of the tunneling probability.

This expression has a form similar to the standard tunneling theories which express the current as a product of the local densities of states of the two systems evaluated at a common point and a difference in the corresponding Fermi functions. Kawasaka et al. ${ }^{50,31}$ who studied the STM current through a Kondo resonance used as a starting point of their considerations the Tersoff and Hamann $\operatorname{expression}^{24}$

$$
I_{e q} \propto \int d \omega\left[f_{t}\left(\omega^{\prime}\right)-f_{s}(\omega)\right] \rho_{t}\left(\omega^{\prime}\right) \rho_{s a}\left(\vec{R}_{t}, \omega\right)
$$

according to which the current at zero temperature is related to the LDOS of the adsorbate plus metal electrons $\rho_{s a}\left(\vec{R}_{t}, \omega\right)$ at the position of the tip, where the local density of states is

$$
\rho_{s a}\left(\vec{R}_{t}, \omega\right)=-\frac{1}{\pi} \operatorname{Im}\left\langle\vec{R}_{t}\left|G^{R}(\omega)\right| \vec{R}_{t}\right\rangle .
$$

The LDOS is expressed in terms of unperturbed metal and adsorbate states by inserting $\sum_{k}|k\rangle\langle k|+| a\rangle\langle a|(\approx 1)$ on both sides of $G$ in (3.13). This is strictly valid only for orthogonal orbitals, $\langle a \mid k\rangle=0$. The four resulting terms, proportional to $G_{a}, G_{k a}, G_{a k}, G_{k k^{\prime}}$, reflect the fact that the LDOS includes both the adsorbate and metal states perturbed by their mutual interaction. Inserting this expansion into Eq. (3.13) gives for $\rho_{s a}\left(\vec{R}_{t}, \omega\right)$

$$
\begin{aligned}
\rho_{s a} & =-\frac{1}{\pi} \operatorname{Im}\left\{\sum_{a}\left|\psi_{a}^{\prime}\right|^{2} G_{a}^{R}+\sum_{k a} \psi_{\vec{k}} G_{k a}^{R} \psi_{a}^{\prime *}+\right. \\
& \left.+\sum_{k a} \psi_{a}^{\prime}\left(\vec{R}_{t}\right) G_{a k}^{R} \psi_{\vec{k}}^{*}+\sum_{k k^{\prime}} \psi_{\vec{k}^{\prime}} G_{k^{\prime} k}^{R} \psi_{\vec{k}}^{*}\right\}
\end{aligned}
$$

where the wavefunctions are evaluated at $\vec{R}_{t}$. The four terms correspond to the terms in the square brackets in (3.4). We can also rewrite $\rho_{s a}$ using the expressions for $G_{a k}$ and $G_{k k^{\prime}}$ in Appendix B. We define $\tilde{\psi}_{a}=\psi_{a}^{\prime}+\sum_{k} \psi_{k} G_{k}^{0} V_{k a}$ and write

$$
\rho_{s a}=-\frac{1}{\pi} \operatorname{Im}\left\{\sum_{k}\left|\psi_{k}\right|^{2} G_{k}^{R}+\sum_{a}\left|\tilde{\psi}_{a}\right|^{2} G_{k a}^{R}\right\} .
$$

Comparison of $(3.15,3.12)$ with $(3.9,3.11)$ shows the difference between our equilibrium limit and the transfer Hamiltonian method ${ }^{2,23}$. The tunneling current and differential conductance in the equilibrium limit provides information about $\tilde{\rho}_{\text {sat }}$ - a local density of states modified by the tunneling matrix elements - rather than the LDOS. In the case when the tunneling takes place into distinct orbitals with different symmetry, $\tilde{\rho}_{\text {sat }}$ can be rather different from $\rho_{s a}$ and the statement that the STM is a measure of local density of states must be understood in this context.

\section{The equilibrium tunneling current and the Fano lineshape}

In this section, we evaluate $\tilde{\rho}_{\text {sat }}$ in Eq. (3.11) and write the equilibrium current using the approximations (2.13), (2.14), and (2.17) for the tunneling matrix elements. We note that the approximations do not require any specification of the substrate electronic structure. The final form of the current allows the discussion of the tunneling resonances in terms of the well established Fano lineshapes.

We introduce new quantities in terms of which the current is expressed. First, we define the "bulk" density-ofstates (DOS) for the substrate and the tip as $\rho_{s}(\omega)=$ $\sum_{k} \delta\left(\omega-\epsilon_{k}\right)$ and $\rho_{t}(\omega)=\sum_{p} \delta\left(\omega-\epsilon_{p}\right)$. The impurity width without the STM tip is defined as 


$$
\Gamma_{a s}\left(\vec{R}_{0}, \omega\right)=2 \pi \rho_{s}(\omega) V_{a}^{2}\left(\vec{R}_{0}\right) .
$$

The adsorbate perturbation on the local density of conduction states at some lateral position between the tip and the adsorbate is discussed in terms of the unperturbed substrate Green's function

$$
G_{0}^{+}\left(\vec{r}, \vec{r}^{\prime} ; \omega\right)=\sum_{k} \frac{\psi_{k}(\vec{r}) \psi_{k}^{*}\left(\vec{r}^{\prime}\right)}{\omega-\epsilon_{k}+i \eta} .
$$

We define two dimensionless quantities related to the real and imaginary parts of the Green's function

$$
\Lambda(\vec{R}, \omega)=e^{Z / \lambda} \frac{\operatorname{Re} G_{0}^{+}(\vec{R}, 0 ; \omega)}{\pi \rho_{s}(\omega)}
$$

and

$$
\gamma(\vec{R}, \omega)=-e^{Z / \lambda} \frac{\operatorname{Im} G_{0}^{+}(\vec{R}, 0 ; \omega)}{\pi \rho_{s}(\omega)} .
$$

These two functions carry the information about both the spatial extent of the metal electron perturbation at arbitrary $\vec{R}$ in the surface region due to a localized perturbation at $\vec{R}_{\|}=0$ and also the spatial resolution of the tip, as we will see later. We have included the exponential factor $e^{Z / \lambda}$ in the definition (3.18), (3.19), and (3.20) because we explicitly take the $k$-independent part of the exponential dependence on position to be part of the tunneling matrix elements $V_{a}\left(Z_{0}\right), t_{a}\left(\vec{R}_{t}, Z_{0}\right)$, and $t_{c}\left(Z_{t}\right)$. We postpone further discussion of $G_{0}^{+}\left(\vec{r}, \vec{r}^{\prime}, \omega\right)$ to the subsection III F.

Finally, we define a dimensionless quantity as the normalized density of the substrate states at a position $\vec{R}$ above the metal surface

$$
\nu(\vec{R}, \omega)=e^{2 Z / \lambda} \frac{\rho_{s}(\vec{R}, \omega)}{\rho_{s}(\omega)}=-e^{2 Z / \lambda} \frac{\operatorname{Im} G_{0}^{+}(\vec{R}, \vec{R} ; \omega)}{\pi \rho_{s}(\omega)} .
$$

The tunneling current $I_{0}$ into a clean metal is given by the first term in Eq. (3.9). The current $I_{0}$ for small bias $\left(V / \phi_{s} \ll 1\right)$ can be written with the above definitions in a familiar form

$$
\begin{aligned}
& I_{0}\left(\vec{R}_{t}, V\right)=\frac{2 e}{h} \int_{-\infty}^{\infty} d \omega \times \\
& \times \rho_{t}\left(\omega^{\prime}\right)\left[f_{t}\left(\omega^{\prime}\right)-f_{s}(\omega)\right] \rho_{s}(\omega) \pi t_{c}^{2}\left(\vec{R}_{t}\right) \nu\left(\vec{R}_{t} ; \omega\right) .
\end{aligned}
$$

Here, $f_{s}(\omega)$ and $f_{t}(\omega)$ are the substrate and STM tip Fermi functions, respectively, and $\omega^{\prime}=\omega-e V$. The tip and substrate are assumed to have common chemical potential $\epsilon_{F s}=\epsilon_{F t}=0$ at zero bias $e V=0$ and we adopt the convention of measuring the energies in the substrateadsorbate complex and in the tip from their respective Fermi levels at finite bias. The bias $V$ is measured with respect to $\epsilon_{F s}$ and is defined as positive when the chemical potential of the tip $\epsilon_{F t}$ is raised. The functions $\rho_{s}$ and $\rho_{t}$ are the substrate and tip densities of states, respectively.

It follows from Eq. (3.21) that the tunneling current $I_{0}$ is independent of temperature if $\rho_{t}, \rho_{s}$, and $\nu\left(\vec{R}_{t}, \omega\right)$ are independent of energy in the relevant energy range. If $\rho_{s}$ shows structure on the scale of the temperature $T$ while $\rho_{t}$ is constant, the current will depend on the temperature of the tip only, and vice versa. These statements are not limited to the case of clean metal surfaces, but also hold for the substrate with an impurity. The same is true for $\mathcal{G}$, the differential conductance.

The equilibrium current in the presence of the adsorbate is written by expressing $\tilde{\rho}_{\text {sat }}$ with the notation and approximations that lead to Eq. (3.21). We define a modified matrix element $\tilde{t}_{a}\left(\vec{R}_{t}, \vec{R}_{0} ; \omega\right)$ for tunneling from tip to the adsorbate state as

$$
\tilde{t}_{a}=t_{a}+\pi t_{c} \rho_{s} \Lambda V_{a} .
$$

The second term represents a coherent process of tipto-surface tunneling, through-surface-propagation, and surface-to-adsorbate hopping. This is completely isomorphic with Fano's coupling of an excited state (here the tip state) with the originally discrete state "modified by admixture of states of the continuum". The reader is enthusiastically directed to the original Fano paper for further enlightenment on this point.

We introduce the Fano ${ }^{29}$ parameter $q\left(\vec{R}_{t}, \vec{R}_{0} ; \omega\right)$ as

$$
q=\frac{\tilde{t}_{a}}{\pi t_{c} V_{a} \rho_{s}} .
$$

We will see later that this definition of $q$ makes the expression for differential conductance formally equivalent with the Fano formula in certain limits. It is rather straightforward now to evaluate $\tilde{\rho}_{\text {sat }}$ using (3.9) and (3.10) in (3.11). After rearranging the terms, we write the current $I_{e q}\left(\vec{R}_{t}, \vec{R}_{0}, \omega\right)$ in the presence of the adsorbate resonance as

$$
\begin{aligned}
& I_{e q}\left(\vec{R}_{t}, \vec{R}_{0}, V\right)=\frac{2 e}{h} \int_{-\infty}^{\infty} d \omega \rho_{t}\left(\omega^{\prime}\right) \times \\
& \times\left[f_{t}\left(\omega^{\prime}\right)-f_{s}(\omega)\right] \rho_{s}(\omega) \pi t_{c}^{2}\left(\vec{R}_{t}\right) Y\left(\vec{R}_{t}, \vec{R}_{0}, \omega\right),
\end{aligned}
$$

with

$$
Y=\nu+\sum_{a} \frac{\Gamma_{a s}}{2}\left\{\left(\gamma^{2}-q^{2}\right) \operatorname{Im} G_{a}^{R}+2 q \gamma \operatorname{Re} G_{a}^{R}\right\} .
$$

In our approximation, the localized nature of the tip and the adsorbate enters through the position dependence of $t_{a}\left(\vec{R}_{t}, Z_{0}\right)$ and the substrate Green's function $G_{0}^{+}\left(\vec{R}_{t}, 0 ; \omega\right)$. The matrix element $t_{a}$ gives an exponentially decreasing amplitude with increasing tip-adsorbate distance and the substrate Green's function gives decreasing amplitude due to the phase difference between electrons entering (or leaving) the surface at the adsorbate site and leaving (or entering) at $\left(\vec{R}_{\|}, z=0\right)$ and also 
due to the exponential decay of the tip wavefunction with increasing $k_{\|}$). We note that, in the wide band limit for the substrate and with the tip near the surface above the adsorbate, $\tilde{t}_{a} \approx t_{a}$, since in this limit $\operatorname{Re} G^{+}$and thus $\Lambda$ vanish.

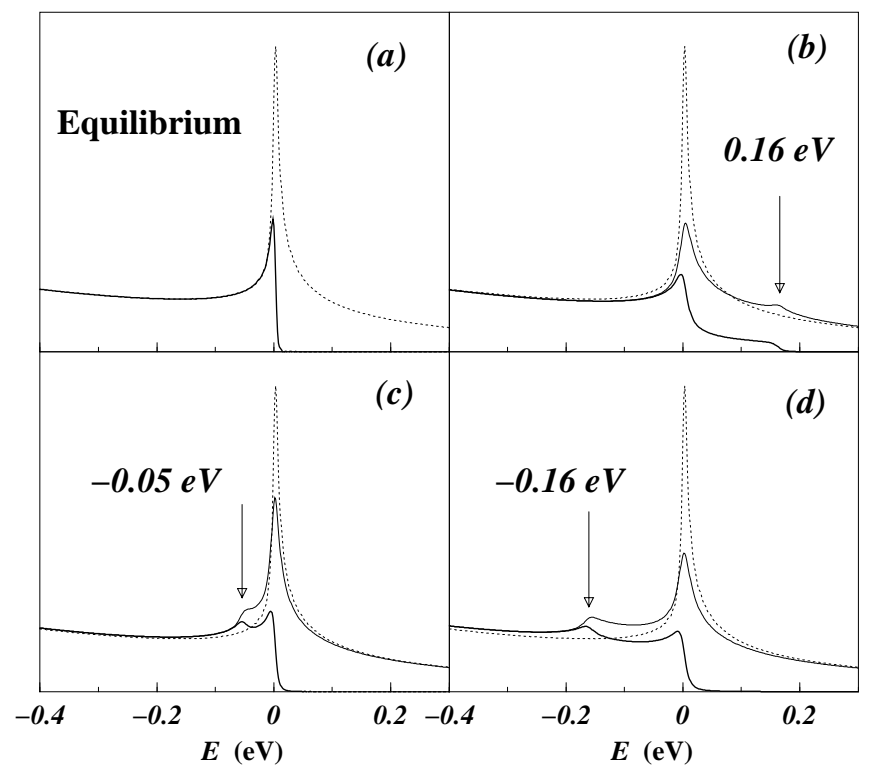

FIG. 2. The spectral function $\rho_{a}(\omega)$ and the occupied density of states of a model Kondo system as a function of bias voltage at small metal-tip separation given by $\Gamma_{a t}=0.1 \Gamma_{a s}$. (a) equilibrium, (b) - (d) finite bias. Bold line $=$ electron population, solid thin $=$ spectral density, dotted $=$ equilibrium spectral density.

\section{Nonequilibrium effects at stronger tip-surface coupling}

We now generalize Eq. (3.24) for the equilibrium tunneling current - obtained in the lowest order in $t_{a p}$ and $t_{k p}-$ by including nonequilibrium effects. The general problem of tunneling for arbitrary relative strength between the tunneling amplitudes $t_{a p}$ and $t_{k p}$ and the hybridization matrix $V_{a k}$ and for finite bias is formulated in Eq. (3.5), but the expression is quite complicated to evaluate in practice. In a typical STM experiment, the tunneling matrix elements $t_{a p}$ and $t_{k p}$ are much smaller than than $V_{a k}$. We can expect the nonequilibrium effects to be important when, at small separations, the magnitude of the two tunneling matrix elements is not a negligible fraction of $\left|V_{a k}\right|$. However, we can always safely assume that $\left|t_{a k}\right|,\left|t_{a p}\right|$ are smaller than $\left|V_{a k}\right|$ in the STM experiments under all realistic conditions.

Therefore we make additional simplifications which are justified by these relations. First of all, we replace $\tilde{V}_{a k}$ by $V_{a k}$ inside Eq. (3.6) and (A5). We neglect the modifications to the tip and substrate wavefunctions, i.e. replace $\tilde{G}_{k k^{\prime}}$ by $\delta_{k k^{\prime}} G_{k}^{0}$ and $\tilde{G}_{p p^{\prime}}$ by $\delta_{p p^{\prime}} G_{p}^{0}$. We also neglect any deviations from thermal electronic distribution in the substrate and tip, i.e. we assume the validity of the fluctuation-dissipation theorem for the tip and substrate Green's functions. On the other hand, when the tipadsorbate coupling is not negligible with respect to the adsorbate-metal hybridization, the current into the resonance can be large enough to produce significant nonequilibrium electronic population on the adsorbate since the time scales for electron dissipation from the resonance into the metal and tip, respectively, are comparable. In this case, the fluctuation-dissipation theorem $G_{a}^{<}=f_{s} \rho_{a}$ is no longer valid for the adsorbate Green's function and we must use the full nonequilibrium $G_{a}^{<}(\omega)$ instead of $f_{s}(\omega) \rho_{a}(\omega)$ in Eq. 3.24.

Under these assumptions, we find it convenient to write the total current with the nonequilibrium effects as $I_{t o t}=$ $I_{e q}+\delta I_{n o n}$, where $I_{e q}$ is formally given by (3.24) and $\delta I_{\text {non }}$ is

$$
\begin{aligned}
\delta I_{\text {non }} & =-\frac{2 e}{h} \sum_{a} \int_{-\infty}^{\infty} d \omega \pi^{2} \rho_{t} \rho_{s}^{2} t_{c}^{2} V_{0}^{2} \times \\
& \times\left(f_{s} \operatorname{Im} G_{a}^{R}+\pi G_{a}^{<}\right)\left(q^{2}+\gamma^{2}\right),
\end{aligned}
$$

where all adsorbate and substrate densities and Green's functions are evaluated at energy $\omega$ and $\rho_{t}$ at $\omega^{\prime}=$ $\omega-e V$. We omitted the spatial arguments for simplicity. The bias dependence enters through the selfconsistent solution of the adsorbate spectral density $\rho_{a}(\omega)=-\frac{1}{\pi} \operatorname{Im} G_{a}^{R}(\omega)$ and the "lesser" Green's function $G_{a}^{<}(\omega)$. In the case of noninteracting system, $(U=0)$, the spectral density does not depend on the bias and the only nonequilibrium (finite bias) effect is given by the difference between the equilibrium $G_{a, e q}^{<}(\omega)=f_{s}(\omega) \rho_{a}(\omega)$ and the nonequilibrium density of occupied states $G_{a}^{<}(\omega)$, as featured in $\delta I_{\text {non }}$.

On the other hand, the spectral density $\rho_{a}(\omega)$ of Kondo systems depends on the bias. This means that $I_{e q}$ also contains nonequilibrium effects and is different from the equilibrium current despite the subscript "eq" and its identical form. The effect of bias on the spectral function depends on the tip hybridization with the discrete impurity level and is similar to that of temperature for $e V \leq T_{K}$ where it broadens the Kondo resonance. At larger biases the broadening increases further and a second peak may develop at the Fermi level of the tip, depending on the strength of the adsorbate-to-tip hybridization $\Gamma_{a t}=2 \pi \rho_{t} t_{a}^{2}$ compared to $\Gamma_{a s}=2 \pi \rho_{s} V_{a}^{2}$ for the relevant impurity orbital. ${ }^{51,52}$ In Fig. 2, we show for different bias voltages the spectral function and electron occupation of the resonance for a model Kondo system with $\Gamma_{a t}$ equal to $\sim 10 \%$ of $\Gamma_{a s}$ and under an additional assumption that $\left|t_{k p}\right| \ll\left|t_{a p}\right|$. The model will be discussed in more detail in section IV B.

\section{E. Differential conductance in the limit of large tip-surface separation}

The differential conductance is obtained directly from (3.24) by differentiating it with respect to the bias, 
i.e. $\mathcal{G}=d I / d V$. We do this here under the assumption that the bias voltage is varied across a sufficiently narrow range so that the density of tip states may be taken constant. Under these assumptions the differential conductance $\mathcal{G}_{e q}$ is

$$
\begin{aligned}
\mathcal{G}_{e q}\left(\vec{R}_{t}, \vec{R}_{0}, V\right) & =\frac{2 e^{2}}{h} \int_{-\infty}^{\infty} d \omega \rho_{t}\left(\omega^{\prime}\right)\left(-\frac{\partial f_{t}\left(\omega^{\prime}\right)}{\partial \omega}\right) \\
& \times \rho_{s}(\omega) \pi t_{c}^{2}\left(\vec{R}_{t}\right) Y\left(\vec{R}_{t}, \vec{R}_{0} ; \omega\right)
\end{aligned}
$$

and for the clean metal

$$
\begin{aligned}
\mathcal{G}_{0}\left(\vec{R}_{t}, V\right) & =\frac{2 e^{2}}{h} \int_{-\infty}^{\infty} d \omega \rho_{t}\left(\omega^{\prime}\right)\left(-\frac{\partial f_{t}\left(\omega^{\prime}\right)}{\partial \omega}\right) \times \\
& \times \rho_{s}(\omega) \pi t_{c}^{2}\left(\vec{R}_{t}\right) \nu\left(\vec{R}_{t}, \omega\right) .
\end{aligned}
$$

These expressions neglect any changes to the tunneling barrier from the finite bias voltage. When these approximation are not justified, the conductance must be obtained by differentiating the expression for current (3.24) and (3.21). This is always the case for $I_{t o t}$ of the previous section when nonequilibrium effects are important.

It is known from the Anderson "compensation theorem" 28 that in the wide band limit $\left(\rho_{s}(\omega)\right.$ is constant and unbounded), the presence of an impurity does not affect the conduction electron density of states at all. However, the density of conduction states is affected locally even in this limit, as can be seen by setting $\psi_{a}=0$ in Eq. (3.14). The perturbation of the conduction electrons is probed by the STM directly if $t_{a}=0$, i.e. when tunneling into the local state is absent due to either large tip-adsorbate separation or because of symmetry. Under these conditions if the STM conductance shows the signature of the local resonance, it is a result of the perturbation of the LDOS of conduction electrons.

An important feature of the final result (3.24) and (3.27) is that the role of the impurity resonance on the tunneling conductance is contained in the Green's function $G_{a}$ of the local adsorbate state. It is then possible to separate the problem into two steps. First, the solutions for the adsorbate and substrate Green's functions are found for a given system; then the tunneling conductance is calculated using the solution in the expression (3.24) or (3.27). Since the equations (3.24) and (3.27) have been obtained under very general assumptions, they can be used as a starting point in the study of a variety of tunneling problems with appropriate approximations for the Green's functions and the tunneling matrix elements. We demonstrate this in the next section, where we first study noninteracting and then Kondo systems. If the approximations to the tunneling matrix elements employed here are too crude, the more general expression (3.5) or (3.11) must be used.

We find that the lineshape $(\mathcal{G}$ vs. $V$ ) of the adsorbate resonance depends sensitively on (1) the relative strength of the tunneling matrix elements $t_{a p}$ and $t_{k p}$, (2) the perturbation of the conduction electron states, (3) the lineshape of the local resonance, i.e. the spectral function $-\frac{1}{\pi} \operatorname{Im} G_{a}$ and $\operatorname{Re} G_{a}$, and on (4) temperature in the case when $T \sim \Gamma$, the width of the resonance. Since the observed perturbation of metal states and the tunneling matrix elements depend on the tip position, so will the lineshape.

\section{F. The substrate Green's function $G_{0}^{+}$and perturbation of the conduction electrons: jellium surface}

There are two ways in which the adsorbate state affects the tunneling conductance: $(\mathrm{A})$ direct tunneling into the discrete state; (B) perturbation of the conduction electron states by the discrete state which consequently contributes to the tip-to-continuum tunneling current. Both contributions drop-off with increasing tip-adsorbate separation. The direct tunneling into the resonance is controlled by $t_{a p}\left(\vec{R}_{t}, Z_{0}\right)$ which is a function of the overlap between the tip and adsorbate wavefunction and thus decays exponentially with the distance. The perturbation of the continuum also vanishes at large distances from the adsorbate. However, its spatial extent shows a more complicated behavior and depends on the details of the electronic structure of the substrate in resonance with the broadened discrete state. It is anticipated that this contribution will show a significantly longer range influence than the decaying exponential, much in the spirit of Friedel oscillations.

The position dependence of the perturbation enters through the Green's function $G_{0}^{+}\left(\vec{R}_{t}, 0 ; \omega\right)$. We note that the imaginary part $\gamma$ appears explicitly in the expression for conductance, (3.27), while the real part $\Lambda$ enters the definition of $\tilde{t}_{a}$. The STM-observable effects of the spatially dependent perturbation of the conduction electrons caused by the local impurity state are thus also controlled by $G_{0}^{+}(3.17)$. In our formulation, a non-trivial $G_{0}^{+}$is intimately related to the dependence of the tunneling element $t_{k p}\left(\vec{R}_{t}\right)$ on the lateral tip position $\vec{R}_{\|}$. With this in mind we focus on the specific problem of tunneling as a function of the tip-adsorbate separation.

We consider a simple approximation for $G_{0}^{+}$based on the assumption that in the relevant surface region the surface corrugations are smoothed out (jellium model) and both the Bloch and/or surface state $\psi_{k}(2.11)$ is given by

$$
\psi_{\vec{k}}(\vec{r}) \propto e^{-\kappa_{s} z} e^{i \vec{k}_{\| \cdot} \cdot \vec{\rho}} .
$$

The states with the smallest $\kappa_{s}$ have the longest tail into the vacuum region and thus will be the most important ones in the tunneling process. These are the states with the smallest $\epsilon_{k_{\|}}$. It is then reasonable to represent $\kappa_{s}$ in terms of the Taylor expansion around the minimum of $\epsilon_{k_{\|}}$with $\epsilon_{k}$ equal to the bias. In most cases, it is reasonable to replace $\epsilon_{k}$ by its Fermi level value. We expand $\epsilon_{k_{\|}}$around its minimum as $\epsilon_{k_{\|}} \approx k_{\|}^{2} / 2 m^{*}$, and 
write $\kappa_{s}=\lambda^{-1}+\lambda k_{\|}^{2} / 2$ plus higher order terms which we neglect. For states far from $\epsilon_{F_{s}}$ and at small tipsurface separation, the expansion should be made around a different value of $\lambda$. For the purpose of this paper, it is sufficient to consider the Fermi level value $\lambda$. We then write

$$
\psi_{\vec{k}}(\vec{r}) \approx e^{-z / \lambda} e^{-\lambda z k_{\|}^{2} / 2} e^{i \vec{k}_{\|} \cdot \vec{\rho}}
$$

As we will show later, the second exponential $e^{-\lambda Z_{t} k_{\|}^{2} / 2}$ is a measure of the tunneling current carrying $k_{\|}$, the property that gives the STM tip its spatial resolution, and the third exponential, $e^{i \vec{k}_{\|} \cdot \vec{R}_{\|}}$, controls the dependence of the tunneling current on the lateral tip position.

With this approximation for Bloch states in the surface region the substrate Green's function (3.17) is

$$
G_{0}^{+}\left(\vec{R}_{t}, 0 ; \omega\right)=e^{-Z_{t} / \lambda} \sum_{k} \frac{e^{-\lambda Z_{t} k_{\|}^{2} / 2} e^{i \vec{k}_{\|} \cdot \vec{R}_{\|}}\left|\psi_{k}(0)\right|^{2}}{\omega-\epsilon_{k}+i \eta} .
$$

For the bulk band state propagation, it is easy to show using Eq. (3.19) that

$$
\gamma\left(\vec{R}_{t}, \omega\right)=\int_{0}^{1} d x J_{0}\left(k_{\omega} R_{\|} \sqrt{1-x^{2}}\right) e^{-\lambda Z_{t} k_{\omega}^{2}\left(1-x^{2}\right) / 2}
$$

and

$$
\Lambda\left(\vec{R}_{t}, \omega\right)=\frac{1}{\pi \rho_{s}(\omega)} \mathcal{P} \int_{0}^{2 D} d \epsilon \rho_{s}(\epsilon) \frac{\gamma\left(\vec{R}_{t}, \epsilon\right)}{\omega-\epsilon},
$$

where $J_{0}$ is the zeroth order Bessel function and $k_{\omega}$ is the wavevector of the substrate state of energy $\omega$. The normalized density of (STM-accessible) conduction states $\nu\left(Z_{t}\right)$ a distance $Z_{t}$ from the surface is

$$
\nu\left(Z_{t}, \omega\right)=\int_{0}^{1} d x e^{-\lambda Z_{t} k_{\omega}^{2}\left(1-x^{2}\right)} .
$$

In calculating $\Lambda, \gamma$, and $\nu$ we assumed jellium-like dispersion relation $\omega=k_{\omega}^{2} / 2 m^{*}$ and use parabolic density of states $\rho_{s}(\omega)=1-\omega^{2} / D^{2}$. The incompatibility of the density of states with the dispersion relation is not important for the purpose of demonstrating the important band structure effects at this level of simplification.

Although the expressions (3.32)-(3.34) are valid for a very simple model of the surface, we believe they contain the most important features of more realistic bulk electronic structures. We now discuss these features beginning with $\gamma$, Eq. (3.32). At large $Z_{t}$, the dominant contribution to the integral in $\gamma$ comes from small values of the argument $y$ in $J_{0}(y)$. In this case, we use the mean value theorem to write $(3.32)$ as $\gamma\left(\vec{R}_{t}, \omega\right)=J_{0}\left(\bar{k} R_{\|}\right) e^{-\lambda Z_{t} \bar{k}^{2} / 2}$ where $\bar{k}=\alpha k_{\omega}$ with $\alpha \in(0,1)$. Clearly, $\alpha \rightarrow 0$ as
$Z_{t} \rightarrow \infty$ and $\gamma\left(\vec{R}_{t}, \omega\right)$ is independent of the lateral tip position. Since at the same time $\Lambda \rightarrow 0$ and $t_{a} \rightarrow 0$, the STM has no spatial resolution in this limit. As the tip moves closer to the surface the spatial resolution increases. In the limit $Z_{t}=0$, the integral in (3.32) can be evaluated and $\gamma\left(\vec{R}_{t}, \omega\right)=j_{0}\left(k_{\omega} R_{\|}\right)$where $j_{0}$ is the spherical Bessel function of zeroth order.

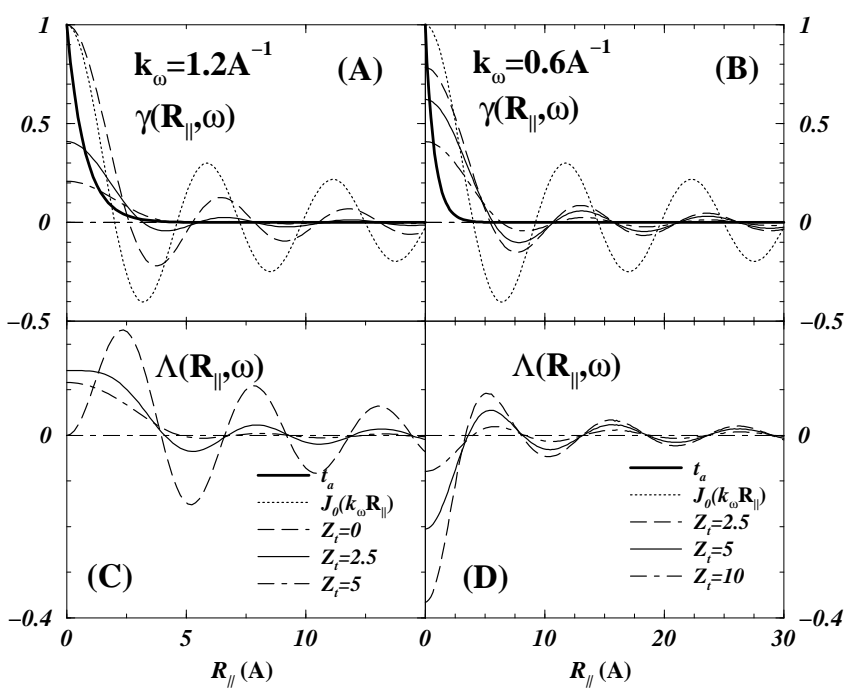

FIG. 3. (A) and (B) show the typical spatial dependence of the tunneling matrix element $t_{a}$ (bold) and of the substrate Green's function $G^{+}$for the jellium model evaluated at $k_{\omega}=1.2 \AA^{-1}$ (left panels) and $k_{\omega}=0.6 \AA^{-1}$ (right panels) for different $Z_{t}$. We parameterize $t_{a} \propto e^{-R_{\|} / a}$ with $a=0.75 \AA$. Also shown is the Bessel function $J_{0}\left(k_{\omega} R_{\|}\right)$for the same $k_{\omega}$, i.e. $\gamma$ of a surface state with $k_{\omega}$. The lower panels $(\mathrm{C})$ and (D) show $\Lambda$, the upper panels (A) and (B) show $\gamma, J_{0}$, and $t_{a}$. The comparison for the two wavevectors assumes identical barrier (damping constant $\lambda$ ).

The resonance lineshape depends on the ratio of the direct tip-adsorbate tunneling amplitude $\left(t_{a}\right)$ to the amplitude for tunneling into the perturbed metal states $\left(t_{c}\right)$ and on the interference of conduction electrons scattering from the impurity. The latter contribution is represented here by the substrate Green's function $G^{+}$. Therefore, the presence of an impurity on the surface can be sensed spectroscopically even if the direct tunneling into the resonance is negligible as is the case, for instance, of $\mathrm{Ce} / \mathrm{Ag}(111)^{22}$. As our estimates for $t_{a}\left(R_{\|}\right)$and $\gamma\left(R_{t}\right)$ indicate, the direct tunneling matrix element $t_{a}\left(R_{\|}\right)$falls off much more rapidly with $R_{\|}$than does $\gamma$. This is due to the limited spatial extent of the tightly bound impurity orbital. Therefore, the relative importance of tunneling into the perturbed continuum is likely to increase with the lateral tip-adsorbate separation.

Using the simple model for $G^{+}$, we show the typical length scales in Fig. 3(A). We plot $t_{a}$ (bold solid line) parameterized as $t_{a} \propto e^{-R_{\|} / \alpha}$ and normalized to one for $R_{\|}=0$ together with $\gamma$ evaluated at three different positions $Z_{t}$ above the surface and with $k_{\omega}=1.2 \AA^{-1}$. 
The exponential fall-off for conduction states at the Fermi level with work functions in the range $4-5 \mathrm{eV}$ would be characterized by $\lambda=0.9 \AA$. The tightly bound discrete state will have larger decay constant and we parameterize it by $\alpha=0.75 \AA$. Clearly, $\gamma$ decays much slower than $t_{a}$ at small tip-surface separations, but the difference in fall-off becomes smaller with increasing $Z_{t}$. We also see by comparison with the Bessel function $J_{0}\left(k_{F} R_{\|}\right)$(light dotted) that the spatial frequency decreases with increasing $Z_{t}$ and the oscillations eventually disappear entirely. This is due to the fact that smaller $k$-vectors have larger weight in the tunneling at greater $Z_{t}$ (see integral (3.32)). The real part $\Lambda$, shown in panel (C) for the same k-vector, has a similar behavior. However, being a Hilbert transform of the imaginary part, the nodes in $\Lambda$ appear at the positions of local extrema of $\gamma$ and vice versa. As we will see later, this property would lead to significant variations in the lineshape with $R_{\|}$if it survived in the real electronic structure. Our results suggest that this is possible only at small $Z_{t}$. We discuss the band structure effects in the following section.

The panels (B) and (D) show the same as (A) and (C) but for smaller wavevector $k_{\omega}=0.6 \AA^{-1}$. Comparison between the right and left thus demonstrates the strong dependence of the substrate Green's function on the wavevector itself, not just the product $k_{\omega} R_{\|}$. The two most significant features are that with decreasing $k_{\omega}$ : (1) the frequency and damping of the oscillations with $R_{\|}$decrease and (2) the dependence on $Z_{t}$ weakens. In comparing the two different energies, we assumed that the damping constant $\lambda$ (i.e. the tunneling barrier) is identical in the two cases. This would be the case in metals with identical work functions for states at the Fermi energy, in one of which the bottom of the band were closer to the Fermi level (smaller $\left.k_{\omega}\right)$. We note that $k_{\omega}=1.2 \AA^{-1}$ corresponds to energy $\omega=5 \mathrm{eV}$ in the middle of the parabolic band with our parameterization. Therefore, the value of $\Lambda\left(\vec{R}_{t}=0\right)=0$ at this energy but is negative for smaller energies, e.g. for $k_{\omega}=0.6 \AA^{-1}$, since in this case there are more high energy continuum states repelling the discrete state downward than low energy states pushing it up. We also see that the value of $\Lambda$ at $R_{\|}=0$ can change sign with $Z_{t}$ depending on the energy $\omega$. We note that, since $\Lambda$ enters the expression for $q$, the Fano parameter could also be negative and the asymmetry of the resonance lineshape could be reversed.

\section{G. Electronic structure effects and the surface states on (111) noble metals}

In the previous section we introduced a simple model of $G^{+}$based on the unperturbed jellium surface. In general, more realistic behavior of $G^{+}$can be obtained from electronic structure calculations. Here we discuss qualitatively the electronic structure effects with special attention to the (111) surfaces of noble metals frequently used in STM studies.

It is well known that (111) surfaces of noble metals contain Shockley surface states inside the projected two dimensional band gap that forms on these surfaces. ${ }^{53,54}$ Both the surface state and bulk wavefunction are given by the same general expression (2.11) outside of the metal surface. However their overall degree of localization at the surface is determined by the position of $\epsilon_{s s}$, the surface state eigenvalue, with respect to the band gap edges. All other things being equal, the most localized surface state occurs when $\epsilon_{s s}$ is at midgap. As $\epsilon_{s s}$ moves towards either band edge, the extension of the evanescent oscillatory tail of the surface state wave function into the bulk increases, ultimately becoming identical to a periodic Bloch function when $\epsilon_{s s}$ hits the band edge. From elementary normalization considerations, surface state extension into the bulk and amplitude at the surface, as reflected in the scale factor (or normalization constant) for the surface state tails (3.29) extending into vacuum, are intimately related; greater population within the bulk means lesser in the surface region. ${ }^{55,56}$ This surface state delocalization into the bulk allows for the local density of bulk states at the surface to greatly exceed that of the surface states, in which case the relative importance of the surface state in the tunneling current will be small near the surface. ${ }^{55}$ However, its importance increases with increasing distance from the surface because the bulk states with shorter wavefunction tails are eliminated from the tunneling. The surface state accounts for about $50 \%$ of the total signal in typical STM tunnel junctions in $\mathrm{Au}(111)^{57,58}$ and is known to be responsible for the interference effects observed on these surfaces near edges, impurities, and in quantum corrals. ${ }^{59-63}$ It will also play a disproportionately important role in the resonance tunneling at large lateral tip-adsorbate distance because its contribution to $G_{0}^{+}$does not decay as quickly as that for the bulk states.

We see the different behavior of the bulk and the surface states in the STM when we consider the propagator $G_{0}^{+}$for the Shockley state. This is again given by Eq. (3.31). However, the k-sum now only extends over the 2-D wavevector $k_{\|}$. Assuming parabolic dispersion for the surface state, $\gamma$ is given by

$$
\gamma\left(\vec{R}_{t}, \omega\right)=J_{0}\left(k_{\omega} R_{\|}\right) e^{-\lambda Z_{t} k_{\omega}^{2} / 2}
$$

and

$$
\Lambda\left(\vec{R}_{t}, \omega\right)=\frac{1}{\pi \rho_{s}(\omega)} \int_{0}^{2 D} d \epsilon \rho_{s}(\epsilon) \frac{\gamma\left(\vec{R}_{t}, \epsilon\right)}{\omega-\epsilon}
$$

where, as before, $k_{\omega}$ is the $2-\mathrm{D}$ wavevector of the substrate state corresponding to energy $\omega$. The contribution of the Shockley state to the normalized density of conduction states $\nu\left(Z_{t}\right)$ is given by

$$
\nu\left(Z_{t}, \omega\right)=e^{-\lambda Z_{t} k_{\omega}^{2}} .
$$

The propagator $G_{0}^{+}$for the surface state is essentially equal to the Bessel function $J_{0}\left(k_{\omega} R_{\|}\right)$weighted by the 
exponential $e^{-\lambda Z_{t} k_{\omega}^{2} / 2}$. Therefore the oscillations are not damped with increasing $Z_{t}$ and only their overall amplitude is diminished. Since the surface state on the noble metal surfaces (111) have a short $k_{F} \sim 0.15-0.2 \AA^{-1}$, its propagator will have a much longer spatial extent than that of the bulk states. The corresponding oscillations thus have a spatial period of about 10 times that of the Bessel function $J_{0}$ in Fig. 3(A) in agreement with the experimental observation of Friedel oscillations. The contribution of the surface state to the total current can carry information about an impurity on the surface over a long distance. The perturbation of the surface state by the impurity should persist over several tens of angströms.

It is also known ${ }^{57}$ that the spectral weight of the surface state decreases near surface imperfections. We expect the same to be true near the adsorbate. While we have explicitly taken into account the interaction of the conduction states with the discrete state " $a$ " through the adsorbate Green's function $G_{a}$, all other adsorbate-metal interactions, such as potential scattering of the conduction electrons from the adsorbate and hybridization of the outer shell adsorbate electronic states with the conduction electrons, are neglected in our model. In principle, these "residual" adsorbate-metal interactions can be included by modifying $G^{+}$and $t_{k p}$. Although a realistic calculation of the system electronic structure is necessary to see the effect of the adsorbate on the behavior of $G^{+}$ around the adsorbate, we believe that it will not produce oscillatory behavior in $G^{+}$. In a typical metal, several bands with anisotropic dispersion relations $\epsilon_{\vec{k}}$ contribute to $G^{+}$giving rise to more complicated behavior with no single frequency. This will further reduce any oscillatory behavior seen in Fig. 3. At larger distance from the adsorbate, the band structure of the clean surface will be reestablished and, as a result, tunneling into the surface state.

Since the importance of the direct tunneling into the tightly bound impurity orbital " $a$ " relative to the tunneling into the metal should be weak and decreases with increasing $R_{\|}$, it is useful to study the asymptotic behavior of the conductance $\mathcal{G}$ in the limit $t_{a}=0$. This is equivalent to replacing the Fano parameter $q\left(\vec{R}_{t}, \vec{R}_{0} ; \omega\right)$ by $\Lambda\left(\vec{R}_{t} ; \omega\right)$ inside $Y\left(\vec{R}_{t}, \vec{R}_{0} ; \omega\right),(3.25)$, in the expression for conductance. It then follows that, if the oscillations in $G^{+}$persist, the lineshape should change with $R_{\|}$and antiresonances should form at positions where $\Lambda^{2}>\gamma^{2}$. Using $G^{+}=\pi \rho_{s} e^{-Z / \lambda}(\Lambda-i \gamma)$ and

$$
\begin{aligned}
& \operatorname{Im}\left\{G^{+}\left(\vec{R}_{t}, \vec{R}_{0}\right) G_{a}^{R} G^{+}\left(\vec{R}_{0}, \vec{R}_{t}\right)\right\}= \\
& -\pi^{2} \rho_{s}^{2} e^{-2 Z_{t} / \lambda}\left\{\left(\gamma^{2}-\Lambda^{2}\right) \operatorname{Im} G_{a}^{R}+2 \Lambda \gamma \operatorname{Re} G_{a}^{R}\right\}
\end{aligned}
$$

we can write $\Delta \mathcal{G}_{e q} \equiv G_{e q}-G_{0}$ at zero temperature by replacing $(-\partial f(\omega) / \partial \omega)$ by $\delta(\omega-V)$ and using $t_{c}(Z)=$ $t_{0} e^{-Z / \lambda}$ as

$$
\Delta \mathcal{G}_{e q}(V)=-\frac{2 e^{2}}{h} t_{0}^{2} \rho_{t}(0) V_{a}^{2} \times
$$

$$
\times \operatorname{Im}\left\{G^{+}\left(\vec{R}_{t}, \vec{R}_{0} ; V\right) G_{a}^{R}(V) G^{+}\left(\vec{R}_{0}, \vec{R}_{t} ; V\right)\right\} .
$$

We see that the resonance in the conductance is a result of an interference between different conduction states scattering resonantly from the impurity. Its long range behavior on the (111) noble metal surfaces is controlled by the surface states. Whether the resonance can be observed at the large distances $(\geq 20 \AA)$ depends on the spectral weight of the surface state and on its hybridization $\left(\sim V_{a}^{2}\right)$ with the impurity orbital " $a$ " (usually $d$ or $f$ ). Interesting spatial effects may be realized in system with suitable boundary conditions. We believe that Eigler's quantum mirage of the Kondo resonance inside the elliptical corral falls into this category ${ }^{64}$ Based on the results of the previous section, we do not expect "Friedel" oscillations at smaller distances and with period of a few angströms characteristic of the bulk k-vector, although we cannot completely rule these out for small $Z_{t}$.

\section{DISCUSSION AND EXAMPLES}

The equations (3.24) and (3.27) were derived under rather general assumptions. They are suitable as a starting point for numerical investigations given the necessary input from electronic structure calculations. In the rest of the paper, we discuss the implications of our theory for several specific cases of interest. In all of these cases we use our simple model for $G^{+}$based on the jellium surface and the DOS given by $\rho_{s}(\omega)=\rho_{t}(\omega)=1-\omega^{2} / D^{2}$ with $D=5 \mathrm{eV}$ the band half width. In order to eliminate the exponential fall-off in the tunneling conductance with the tip-surface separation and the background distortions, we plot the normalized change in conductance due to the additional impurity defined as

$$
\Delta \mathcal{G}_{e q}(V) \equiv h\left(\mathcal{G}_{e q}(V)-\mathcal{G}_{0}(V)\right) /\left(2 \pi e^{2} \rho_{t}(0) t_{c}^{2}\right),
$$

where $\mathcal{G}_{e q}$ is given by (3.27) and $\mathcal{G}_{0}$ by (3.28). This is equivalent to replacing $Y$ by $\Delta Y=Y-\nu$ in the expression for $\mathcal{G}$.

Although we were motivated by the experimental observation of the Kondo resonance ${ }^{21,22}$ and this work is mostly applied to the tunneling through the Kondo resonance, we discuss many of the tunneling properties on the simple noninteracting model. We do this primarily because most of the STM observable characteristics of the Fano resonance are common to the single particle and Kondo resonances, despite the difference in processes that give rise to the two resonances. We wish to point out these general features on a model that is conceptually far simpler and more familiar to the surface science community than the Kondo model, and emphasize that the resonances can also be observed in systems with nonmagnetic impurities with a tightly bound orbital near the Fermi level. Finally, the connection with Fano result and the consequences of the spatial resolution of the STM become more transparent when the same noninteracting Anderson Hamiltonian is used. 


\section{A. Noninteracting adsorbate}

We begin our discussion with an adsorbate-metal system described by the non-interacting Anderson model $(U=0)$. The impurity resonance is characterized by its energy $\epsilon_{0}$ and the width $\Gamma_{a s}$. The retarded Green's function $G_{a}^{R}(\omega)$ for the adsorbate state is

$$
G_{a}^{R}=\left(\omega-\epsilon_{0}-\operatorname{Re} \Sigma_{a}+i \Gamma_{a s} / 2\right)^{-1}
$$

where $\Gamma_{a s}\left(Z_{0}, \omega\right)$ is defined in Eq. (3.16) and $\operatorname{Re} \Sigma_{a}\left(Z_{0}, \omega\right)=\mathcal{P} \sum_{k}\left|V_{a k}\left(Z_{0}\right)\right|^{2}\left(\omega-\epsilon_{k}\right)^{-1}$ is the real part of the self-energy for the noninteracting Anderson model (not to be confused with the real part of the substrate Green's function $\Lambda$ given in Eq. (3.18)). Following Fano we now define the dimensionless energy parameter $\epsilon\left(\vec{R}_{0}, \omega\right)$ by

$$
\epsilon=\frac{2\left(\omega-\epsilon_{0}-\operatorname{Re} \Sigma_{a}\right)}{\Gamma_{a s}} .
$$

We neglect all nonequilibrium effects since they are likely to be insignificant for the noninteracting system under most experimentally realizable conditions. The differential conductance, in lowest order in $t_{c}$ and $t_{a}$, is given by Eq. (3.27) where $Y$ for the noninteracting system takes the form

$$
Y \equiv Y_{0}=\nu+\frac{q^{2}-\gamma^{2}+2 \epsilon \gamma q}{1+\epsilon^{2}}
$$

All terms are evaluated at energy $\omega$ and at the appropriate tip position. We note that $Y_{0}(0,0, \omega) \equiv Y_{00}$ characterizing the unphysical case of the STM tip in contact with surface at the position of the adsorbate (embedded in the surface) has the analytic form obtained by Fano

$$
Y_{00}=\frac{(q+\epsilon)^{2}}{1+\epsilon^{2}}
$$

although the inherent energy dependence of $q$ (through $\Lambda(\omega))$ could distort the pure Fano character of the lineshape, even for this "almost atomic physics" STM example. At all other tip positions, the shape of the resonance will be described by the more general expression (4.4), which is equivalent to a sum of a "Lorentzian" plus a "Fano" profile,

$$
Y_{0}=\frac{\nu^{\prime}}{1+\epsilon^{2}}+\nu \frac{\left(q^{\prime}+\epsilon\right)^{2}}{1+\epsilon^{2}}
$$

with $\nu^{\prime} \equiv \nu+q^{2}-\gamma^{2}-q^{2} \gamma^{2} / \nu$ and $q^{\prime} \equiv q \gamma / \nu$. Decomposition of $Y_{0}$ in this manner may be useful when analyzing experimental lineshapes. Note that the additional intrinsic energy dependences of $\nu$ and $\gamma$ could further distort the standard lineshape.

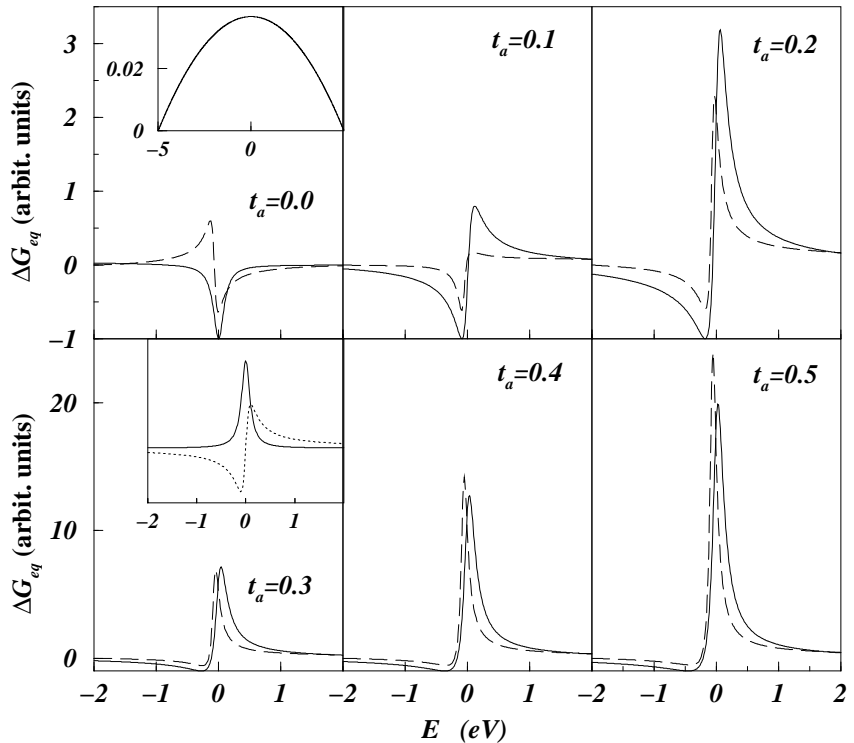

FIG. 4. Differential conductance $\Delta \mathcal{G}_{e q}$ as a function of the strength of the direct tunneling matrix $t_{a}$ (in units of $t_{c}$ ). The model parameterization is given in text. The two curves correspond to two different impurity level energies $\epsilon_{0}=5 \mathrm{eV}$ (solid) and $2 \mathrm{eV}$ (dashed) from the bottom of a symmetric band $10 \mathrm{eV}$ wide. The two insets show the model density of states $\rho_{s}$ for the conduction electrons (upper panel) and the imaginary (solid) and real (dashed) part of $G_{a}(\omega)$ (lower panel).

\section{Lineshape dependence on electronic structure and on the relative strength of $t_{a}$ and $t_{c}$}

We first show (Fig. 4) the dependence of $\Delta \mathcal{G}_{e q}$ on the ratio $\left(t_{a} / t_{c}\right)$ for $\vec{R}_{t}=0$ and a resonance at the Fermi level. The solid line corresponds to a resonance at the center of a parabolic band (symmetric around its center), i.e. $\epsilon_{0}=5 \mathrm{eV}$ from the bottom of the band and the dashed line corresponds to a resonance at $\epsilon_{0}=2 \mathrm{eV}$ from the bottom of the band. The two energies correspond to the band energy $\epsilon_{k}$ with wavevector $k=1.2 \AA^{-1}$ and $k=0.6 \AA^{-1}$ in our jellium model (Fig. 3). The resonance width is $\Gamma_{a s}=0.2 \mathrm{eV}$ in both cases. At zero temperature, from Eq. (3.27) and (4.4) we write

$$
\mathcal{G}_{e q}(V)=\frac{2 e^{2}}{h} \rho_{s}(V) \pi \rho_{t}(0) t_{c}^{2} Y_{0}(V)
$$

In order to make connection with the Fano result, we plot the conductance for small tip-metal separation with the tip above the adsorbate $\left(\vec{R}_{t}=0\right)$. The lineshape $Y_{0}$ is then given by the Fano formula (4.5). The plotted quantity $\Delta \mathcal{G}_{e q}(V)$ in Fig. 4 is then given by

$$
\Delta \mathcal{G}_{e q}(V)=\rho_{s}(V)\left(\frac{(q+\epsilon)^{2}}{1+\epsilon^{2}}-1\right) .
$$

The Fano parameter $q$ depends not only on the ratio $\left(t_{a} / t_{c}\right)$ but also on energy and electronic structure. We 
see this most clearly in the first panel where $t_{a}=0$. The resonance placed at the center of the band produces a symmetric dip in $\Delta \mathcal{G}$ characteristic of $q=0$, whereas the resonance at $\epsilon_{0}=2 \mathrm{eV}$ has an asymmetric lineshape due to the negative contribution from $\Lambda$ to $q$ (see Fig. 3). Its lineshape actually becomes symmetric at finite value of $t_{a}$. The value of $t_{a}$ inside each panel is given in units of $t_{c}$. The inset in the upper panel shows the model density of conduction states $\rho_{s}$ and the lower panel inset shows the spectral function $\rho_{a}=-\frac{1}{\pi} \operatorname{Im} G_{a}^{R}$ (solid) and $\operatorname{Re} G_{a}^{R} / \pi$ (dotted) for the level at the center of the band.

As the strength of the direct tunneling $t_{a}$ increases with respect to $t_{c}$, the resonance develops its characteristic asymmetric shape and, eventually, at large $t_{a} / t_{c} \gg 1$ it acquires the shape nearly indistinguishable from that of the impurity spectral function $\rho_{a}(\omega)$. With increasing tip-adsorbate separation, the signal from the resonance must disappear as both the tunneling element $t_{a}$ and $G^{+}\left(\vec{R}_{t}, \omega\right)$ tend to zero. The differential conductance is then determined by the density of states of the clean surface. This property is not present in the Fano expression. We now discuss this behavior.

\section{Lineshape dependence on the tip-surface separation}

Using the same model system as in the previous section with the resonance at the center of the band $\left(\epsilon_{0}=5 \mathrm{eV}\right.$ from the bottom of the band), we demonstrate the dependence on $Z_{t}$ (with $\vec{R}_{\|}=0$ ) in Fig. 5 . We make the following model for the tunneling matrix element $t_{a}\left(\vec{R}_{t}, \vec{R}_{0}\right)$ and $t_{c}\left(\vec{R}_{t}\right)$. The exponential fall-off of the metal and adsorbate wavefunctions is controlled by different decay constants. The adsorbate state $\psi_{a}$ is tightly bound especially for narrow resonances of interest here. The conduction electron wavefunctions, on the other hand, typically belong to the outer $s$ or $p$ orbitals and have longer tails into the vacuum. As a consequence, the ratio $t_{a} / t_{c}$, and thus also the Fano parameter $q$, changes with $Z_{t}$. In order to incorporate this property, we use the matrix elements (2.17) and $t_{c}=t_{0} e^{-Z_{t} / \lambda}$, where $\alpha=0.75 \AA$, $\lambda \approx 0.9 \AA, t_{0}=25 \mathrm{meV}$, and $t_{a}=0.1 t_{c}$ at $Z_{t}=2 \AA$. Under these conditions, the $q$ parameter tends to zero with increasing $Z_{t}$.

The panel (a) shows the normalized $\Delta \mathcal{G}_{e q}$ for this model. The lineshape undergoes only moderate changes with $Z_{t}$ within the experimentally relevant range. We expect this to be a general property. In order to understand the behavior, we discuss the lineshape dependence on tipsurface separation conceptually in terms of two contributions: (1) different decay constants for the discrete $\psi_{a}$ and metal $\psi_{k}$ states at the Fermi level, and (2) different decay constant for metal states at $\epsilon_{F s}$ with different $k_{\|}$. We separate the observable consequences of these two effects in panels (b) and (c). The first contribution produces changes in $q$ due to the changing relative strength between $t_{a}$ and $t_{c}$. We demonstrate this in Fig. 5(b) where only this contribution is taken into account by setting $Z_{t}=0$ inside the substrate Green's function $G^{+}$, or equivalently by setting $\gamma=\nu=1$ and $\Lambda=0$ in Eq. (4.4). This limit does not correspond to a real situation and does not lead to the correct $Z_{t} \rightarrow \infty$ limit. It is shown here merely as an example of the contribution (1) to the $Z_{t}$ dependence of the tunneling conductance. With our parameterization, this case is identical with $q$ changing from $q \approx 0.8$ at $Z_{t}=3 \AA$ to $q \approx 0.2$ at $Z_{t}=9 \AA$. As $Z_{t}$ increases further, $q \rightarrow 0$ and the resonance becomes symmetric. However, we see that the normalized conductance $\Delta \mathcal{G}_{e q}$ does not vanish in the limit $Z_{t} \rightarrow \infty$.

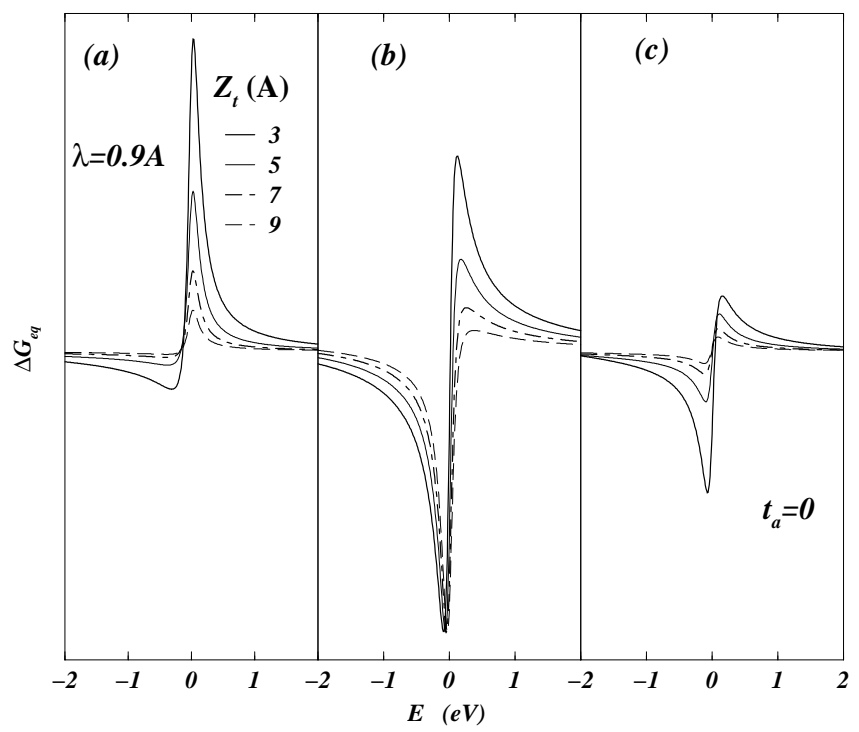

FIG. 5. Differential conductance $\Delta \mathcal{G}_{e q}$ vs. $Z_{t}$ for a model described in the text. (a) the complete dependence on the tip distance $Z_{t}$; (b) assumes $Z_{t}=0$ inside $G^{+}$and thus neglects the wavevector dependent effects; (c) is same as (a) but with $t_{a}=0$, i.e. it only includes the effect of decreasing spatial sensitivity incorporated through the substrate Green's function $G^{+}$. The vertical scale is arbitrary but identical in all panels.

Fig 5(c) takes the second contribution (2) into account while leaving out the first one. We chose $t_{a} / t_{c}=0=$ constant, which would be the case if there were no direct tunneling into the discrete state. In this extreme limit, any changes in lineshape are a consequence of the varying weight that different $k_{\|}$metal states play in the tunneling at different $Z_{t}$. This occurs because the $k_{\|}=0$ metal wavefunctions given by Eq. (3.30) have the greatest extension into the vacuum and as a result the spatial resolution of the tip decreases. Therefore the signature of the resonance in $\mathcal{G}$ decreases even after normalization of the current for different $Z_{t}$ as the ratio $\Delta Y / \nu \rightarrow 0$ with $Z_{t} \rightarrow \infty$. Fig $5($ a) shows the combined effect of the contribution and represents realistic conditions. It accounts correctly for the changing lineshape, as well as its disappearance. We again emphasize that realistic band structure is desirable for making quantitative statements. 
Finally, we comment on the experimental issues. It is clear that the lineshape dependence on $Z_{t}$ will be observable only if it can be studied over a reasonably large range of $Z_{t}$, this being limited by the experimental resolution and detection capabilities. The most favorable case is one in which the direct tunneling $t_{a}$ into the resonance is strong at small $Z_{t}$, i.e. $q$ is large, and the difference in relevant decay constants for the adsorbate and metal electrons, $a$ and $\lambda$ respectively, is large. This is not the case in the experiments ${ }^{21,22}$ where $q$ is small. Therefore, we do not expect significant changes in the lineshape with $Z_{t}$ in these systems. Since our model is based on realistic parameterization, we expect the behavior shown in Fig. 5 to serve as a guide for order of magnitude estimates for the spectral dependence on $Z_{t}$.

The direct effect of the STM tip on the system and thus also on the lineshapes is not taken into account here. This issue is discussed in section IV B 3.

\section{Lineshape dependence on the lateral tip position}

As we already discussed in section III F, the resonance lineshape depends on the relation between the spatial dependence of the direct tunneling and the propagation of the adsorbate-induced perturbation through the metal. Fig. 3 shows that the direct tunneling into the resonance $\left(t_{a}\right)$ is expected to fall off faster than the perturbation. Therefore, at large $R_{\|}$, the lineshape will be given by the $t_{a}=0$ limit conductance. We show the dependence of $\Delta \mathcal{G}_{e q}$ on $R_{\|}$in Fig. 6. We do this again for the model described in the previous section (Fig. 5) with $t_{a}=0$ and a resonance at the center of the band, i.e. $\epsilon_{0}=5 \mathrm{eV}$ from the bottom of the band which corresponds to $k_{\omega}=$ $1.2 \AA^{-1}$ in Fig. 3. The solid line corresponds to $Z_{t}=5 \AA$ and the dashed line to $Z_{t}=0 \AA$.

The unphysical case of $Z_{t}=0$ (dashed) is shown to emphasize the possible consequences of the oscillations in $G_{0}^{+}$displayed in Fig. 3. We chose the lateral tip positions in the figure to coincide with the nodes and zeros of $\Lambda$ and $\gamma$ to show the dramatic changes in the lineshape with $R_{\|}$due to the oscillations in $\Lambda$ and $\gamma$. Since the spatial decay of the oscillations is small at $Z_{t}=0$, the sequence of resonances and antiresonances appear in the range $R_{\|} \in(0,10) \AA$. The possibility for such antiresonances is discussed implicitly in the work of Kawasaka ${ }^{31}$ and explicitly by Schiller and Hershfield ${ }^{32}$. However, this behavior is not observed in the experiments by Madhavan et $a .^{21}$ and $\mathrm{Li}$ et $a{ }^{2} .^{22}$ due to the smoothing of the electronic structure with increasing distance from the surface that we discussed in III F.

In fact we would not expect the dramatic variations in line shape with $R_{\|}$reported by Schiller and Hershfield ${ }^{32}$ to be observed. The reason is apparent from the behavior of $G^{+}$as a function of $Z_{t}$ (Fig. 3). As the tip distance from the surface increases the oscillations are destroyed by the increasing weight of the lower frequency (small $k_{\|}$) components at larger $Z_{t}$ interfering destructively with those given by $k_{\omega}$. For this value of $k_{\omega}$, the oscillations are effectively damped when $Z_{t} \geq 5 \AA$ and the shape of the resonance does not change significantly as shown by the bold line in Fig. 6. We expect that band structure effects will suppress the oscillations even further.

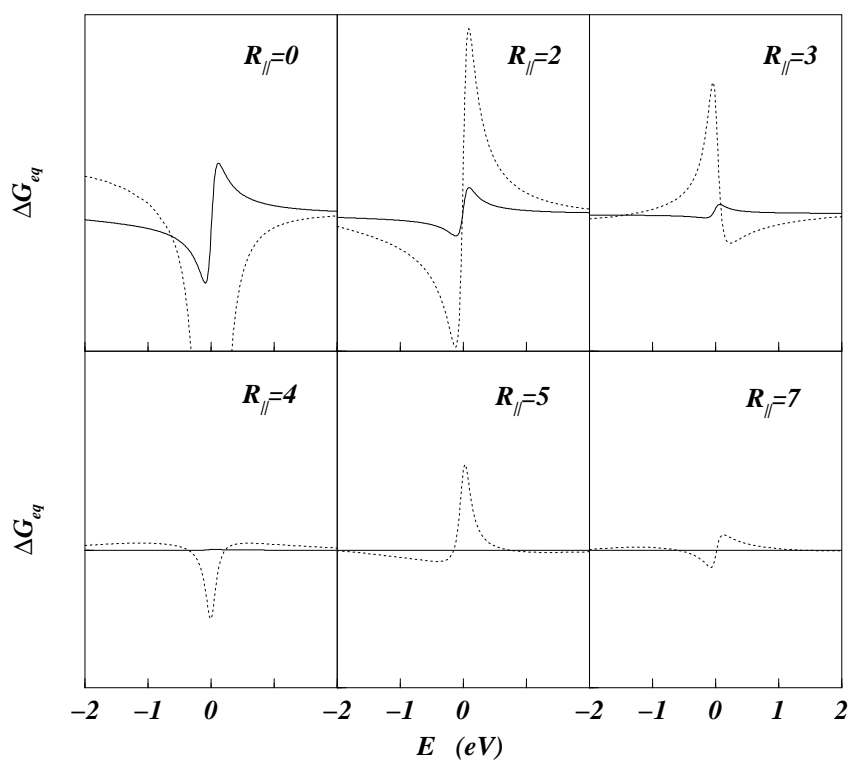

FIG. 6. The normalized differential conductance $\Delta \mathcal{G}_{e q}$ as a function of the lateral tip position $R_{\|}$for the same model as in Fig. 5: $Z_{t}=5 \AA$ (solid) and $Z_{t}=0 \AA$ (dotted). The contribution of the surface states to $G_{0}^{+}$is not included here.

We also find that the spatial extent of the resonance in the spectrum should decrease as the STM is retracted, as long as the signal is due to the bulk states. At $Z_{t}=0 \AA$, the resonance is still visible at $R_{\|} \sim 10 \AA$ but only to about $R_{\|} \sim 4 \AA$ at $Z_{t}=5 \AA$. This is a somewhat shorter distance than that found experimentally for $C o / A u(111)$ and $\mathrm{Ce} / \mathrm{Ag}(111)^{21,22}$. Although the Fermi wavevector $k_{F} \approx 1.2 \AA^{-1}$ used in Fig. 6 is close to the free electron value of $k_{F}$ for the noble metals, the disagreement is not surprising since we made no real attempt at realistic electronic structure description. Smaller values of $k_{F}$ would increase the spatial extent as would smaller values of $Z_{t}$ and $\lambda$.

Interestingly, the $Z_{t}=0$ [dotted] lineshape progression shown in Fig. 6 is qualitatively similar to the family of lineshapes that would be expected from surface state propagation, but with $R_{\|}$, the lateral tip-adatom separation rescaled upward by nearly an order of magnitude. This claim is based on the qualitative similarity between the bulk $G_{0}^{+}$at $Z_{t}=0$ and the surface $G_{0}^{+}$. The bulk $\gamma\left(R_{\|}, \epsilon_{F}\right)=j_{0}\left(k_{F} R_{\|}\right)$[the dashed curve in Fig. 3] at $Z_{t}=0$ the analogous surface state $\gamma\left(R_{\|}, \epsilon_{F}\right)=J_{0}\left(k_{F} R_{\|}\right)$ [dotted curve in Fig. 3] both exhibit long range oscillations unlike the bulk state at $Z_{t} \geq 5 \AA$. However since $k_{F} \sim(0.1-0.2) \AA^{-1}$ for the surface state band, $J_{0}\left(k_{F} R_{\|}\right)$ shown in Fig. $3(\mathrm{~A})$ for $k_{F}=1.2 \AA^{-1}$ should be plot- 
ted with this smaller $k_{F}$ when referring to actual noble metal surface state bands, in which case the observable $R_{\|}$-dependent lineshape evolution in Fig. 6 would still be representative, but with $R_{\|}$rescaled by the factor $1.2 / 0.15=8$. From this it is easy to appreciate that the dramatic lineshape variations will occur mainly at very large lateral separations. Clearly, realistic electronic structure calculations are necessary to answer the more quantitative questions.

At large values of $R_{\|}$and for broader resonances, an additional mechanism for distortion of the lineshape is possible if the relative change in the length of $k_{\omega}$ in the energy range given by the resonance width $(\Gamma)$ near $\epsilon_{0}$ is large. In principle, this gives rise to the possibility of the oscillatory behavior of $J_{0}$ with respect to energy exhibiting itself in the spectrum. However, the conditions for this effect in $\mathcal{G}(\omega)$ would require the relative change $\Delta k_{\omega} / k_{\epsilon} \approx \sqrt{1+\Gamma / 2 \epsilon_{0}}-\sqrt{1-\Gamma / 2 \epsilon_{0}}$ to be $>2 \pi / k_{\epsilon} R_{\|}$. In this case, the argument of the Bessel function in (3.32) will vary over several periods starting at a small value at the bottom of the resonance. It is however clear that the width of the resonance and the distance from the impurity would have to be much larger than in the recent experiments with Kondo impurities. Moreover, these oscillations could only be observed through the surface state due to the damping of oscillations in the bulk.
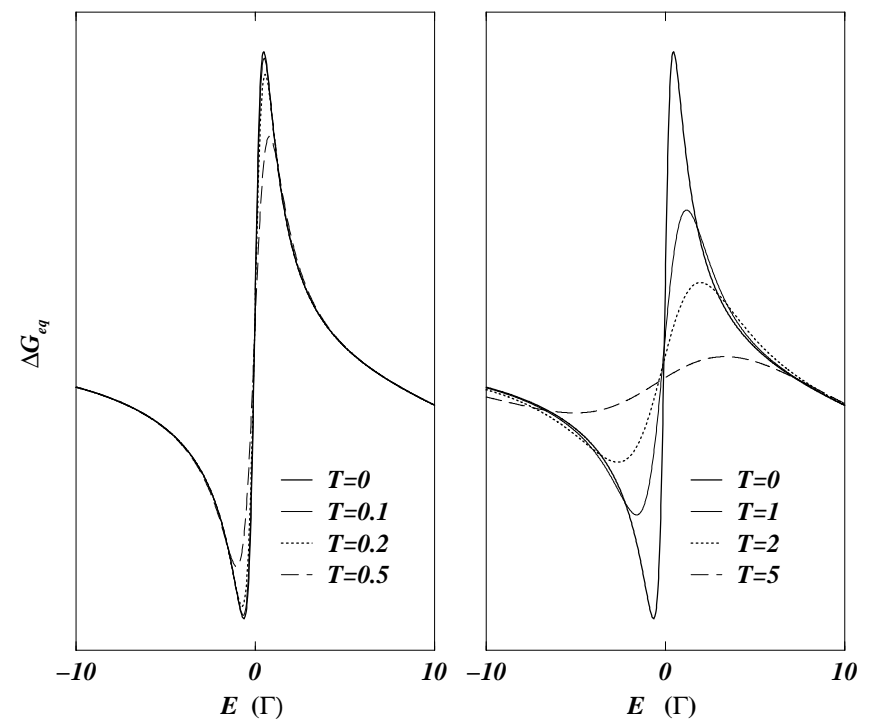

FIG. 7. $\Delta \mathcal{G}_{e q}$ as a function of temperature for $Z_{t}=5 \AA$ and the same system as in Fig 5. Temperature and energy are given in units of the resonance width, $\Gamma$.

\section{Temperature dependence of differential conductance}

The temperature dependence of the tunneling conductance through a narrow resonance is an important issue especially in considerations of tunneling through the Kondo resonance which itself is temperature dependent. We revisit this issue in the section IV B 2 on Kondo ef- fect. Here, we demonstrate the effect of the broadening in the Fermi function on the spectrum when the impurity spectral function is independent of temperature.

We assume the density of tip states and the substrate conduction electrons to be constant on the scale of the width $\Gamma$ near the tip Fermi level and around the resonance. The temperature dependence of the conductance then follows from equation (3.27) with (4.4). We consider only the case of the tip directly above the adsorbate for simplicity and write

$$
\mathcal{G}(V)=\frac{2 e^{2}}{h} \pi \rho_{t} \rho_{s} \int_{-\infty}^{\infty} d \omega\left(-\frac{\partial f_{t}\left(\omega^{\prime}\right)}{\partial \omega}\right) t_{c}^{2} Y_{0}\left(\vec{R}_{t}, \omega\right)
$$

It follows from this expression that the differential conductance only depends on the temperature of the STM tip. This is a consequence of the assumption that the density of tip states is constant in the relevant energy range and the tunneling barrier is not modified by the bias. The differential conductance is then a function of the spectral density of the substrate states and is independent of their occupation. Generally, if the DOS in the STM tip varies significantly on energies $\sim \Gamma_{a s}$, the substrate temperature would also enter. If the density of states in both the substrate and the tip were constant, no temperature dependence would be observed. It follows from Eq. (4.9) that the temperature $T$ must be $\sim \Gamma$, in order to have a significant effect on the conductance.

We show the temperature dependent $\mathcal{G}_{\text {eq }}$ in Fig. 7. The spectral density $\rho_{a}(\omega)$ with the narrow resonance at the Fermi level (the same system parameters as in Fig. 4) is independent of $T$. The STM tip is directly above the resonance with $Z_{t}=5 \AA$. In the range $T \leq 0.1 \Gamma$, no temperature dependence is noticeable. However, when $T \sim(0.2-0.5) \Gamma_{a s}$ the differential conductance shows a rather strong dependence on temperature, and at $T \gg \Gamma$ the sensitivity of the STM to the resonance disappears. The temperature dependence in Fig. 7 comes entirely from the broadening of the Fermi function of the tip.

\section{B. Tunneling into Kondo resonance}

In the previous section IV A, we discussed the STM conductance in tunneling through a noninteracting impurity $[U=0$ in Hamiltonian (2.1)], frequently referred to as the resonant level model (RLM). We now turn to the case of magnetic impurities and tunneling through a Kondo resonance. We begin with the case of a weak tip-metal coupling. However, for the Kondo systems this assumption is more restrictive than for the RLM model, and for this reason, we later take advantage of our nonequilibrium approach to account for the direct effect of the tip on the impurity spectral density, while still neglecting the tip's effect on the metal states. 


\section{Conceptual and theoretical approach}

Since our earlier derivation of the current and conductance is valid for arbitrary interaction $[U \neq 0$ in Eq. (2.1)], the final results (3.24) and (3.27) also hold in the Kondo and mixed-valent regimes of the Anderson model (i.e. $U \gg \Delta$ ). The properties of the adsorbate enter through the Green's function $G_{a}$. The problem is thus reduced to finding the one electron Green's function $G_{a}$.

However, we first consider the tunneling for a spin $1 / 2$ $(a \equiv \sigma)$ impurity in the Kondo limit, $\left(\epsilon_{F s}-\epsilon_{0}\right) \gg \Gamma$ and $\left(\epsilon_{0}-\epsilon_{F s}+U\right) \gg \Gamma$. The Kondo resonance has a very small weight and is due to spin fluctuations. The possible tunneling channels in this case are shown in Fig. 8 as processes (1) and (3). The system can be described by the Kondo Hamiltonian in this limit

$$
\begin{aligned}
H_{s}\left(Z_{0}\right) & =\sum_{k \sigma} \epsilon_{k} c_{k \sigma}^{\dagger} c_{k \sigma}+\sum_{p \sigma} \epsilon_{p} c_{p \sigma}^{\dagger} c_{p \sigma}+ \\
& +\sum_{k p \sigma}\left\{t_{k p}\left(\vec{R}_{t}\right) c_{k \sigma}^{\dagger} c_{p \sigma}+\text { H.c. }\right\}+ \\
& +J_{s} \sum_{k k^{\prime} \sigma \sigma^{\prime}}\left(c_{k \sigma^{\dagger}}^{\dagger} \vec{s}_{\sigma \sigma^{\prime}} c_{k^{\prime} \sigma^{\prime}}\right) \cdot \vec{S}+ \\
& +J_{t} \sum_{p p^{\prime} \sigma \sigma^{\prime}}\left(c_{p \sigma}^{\dagger} \vec{s}_{\sigma \sigma^{\prime}} c_{p^{\prime} \sigma^{\prime}}\right) \cdot \vec{S}+ \\
& +J_{s t} \sum_{k p \sigma \sigma^{\prime}}\left\{\left(c_{k \sigma}^{\dagger} \vec{s}_{\sigma \sigma^{\prime}} c_{p \sigma^{\prime}}\right) \cdot \vec{S}+H . c .\right\}
\end{aligned}
$$

where the first three terms were also present in the total Hamiltonian introduced in section II and describe the unperturbed metal and tip states and the coupling between the two. The remaining terms give rise to spin fluctuations in the presence of the magnetic impurity. The terms with couplings $J_{s}$ and $J_{t}$ correspond to the exchange interaction of the local spin with the substrate and tip electrons, respectively. The last term $\left(J_{s t}\right)$ corresponds to the effective tip-substrate exchange interaction in which charge is transported between the tip and the surface. This Hamiltonian can be obtained from $H_{t o t}$ of section II using the Schrieffer-Wolf transformation which relates $J_{s}, J_{t}$ and $J_{s t}$ to $V_{a}$ and $t_{a}$. For the symmetric Anderson model, $J_{s}=4 V_{0}^{2} / U, J_{s t}=4 t_{a} V_{a} / U$, and $J_{t}=4 t_{a}^{2} / U$. Using the continuity equation (3.1), the current is

$$
\begin{aligned}
I & =\frac{2 e}{\hbar} \operatorname{Im}\left\{\sum_{k p \sigma} t_{k p}\left\langle c_{k \sigma}^{\dagger} c_{p \sigma}\right\rangle+J_{s t} \sum_{k p \sigma \sigma^{\prime}}\left\langle c_{k \sigma}^{\dagger} \vec{s}_{\sigma \sigma^{\prime}} c_{p \sigma^{\prime}} \cdot \vec{S}\right\rangle+\right. \\
& \left.+J_{t} \sum_{p p^{\prime} \sigma \sigma^{\prime}}\left\langle c_{p \sigma}^{\dagger} \vec{s}_{\sigma \sigma^{\prime}} c_{p^{\prime} \sigma^{\prime}} \cdot \vec{S}\right\rangle\right\} .
\end{aligned}
$$

The first term is identical with the first term in Eq. (3.3). In the lowest order of the tip-system couplings $\left(t_{k p}, J_{s t}, J_{t}\right)$, the third term does not contribute. The first term corresponds to the direct tip-substrate tunneling channel - process (1) in Fig. 8 - which includes the scattering of conduction electrons from the local moment. The second term corresponds to the direct tunneling into the magnetic impurity - process (3) in Fig. 8. We note that the spin flip scattering that gives rise to the Kondo effect is a higher order process. In the lowest order, the channel (1) and the spin-flip component of (3) do not give rise to interference because the final states have different spin states. The lowest spin-flip process that does interfere with (1) is of second order in $J$ and proportional to $J_{s} J_{s t}$.

In the limit of large tip-metal separation, equivalent to the condition $\left(J_{s} \gg J_{s t} \gg J_{t}\right)$, the third term in Eq. (4.11) as well as higher order contributions from $J_{s t}$ are neglected and all other exchange processes are included in principle. This is equivalent to assuming that the state of the metal-adsorbate system is determined only by $J_{s}$ and is unaffected by the presence of the tip. Theoretically, the problem then reduces to finding the spectral properties of the system without the tip and using them in the expansion for tunneling via the two terms in Eq. (4.11).

As the system parameters move away from the Kondo limit - that is either $\epsilon_{0}$ shifts towards $\epsilon_{F s}$ or $U$ becomes smaller - valence fluctuations appear. The Kondo resonance is then due to both the spin and charge fluctuations. The separate energy scale due to the spin fluctuations eventually disappears in the mixed-valent regime and the Kondo peak merges with the broad resonance centered at $\epsilon_{0}$. In the intermediate regime, where both charge and spin fluctuations coexist on the impurity, another tunneling channel exists. This channel is denoted by (2) in Fig. 8. It also includes the contribution from higher order non-flip processes similar to (3). We study the system in this regime with the Hamiltonian defined in section II.

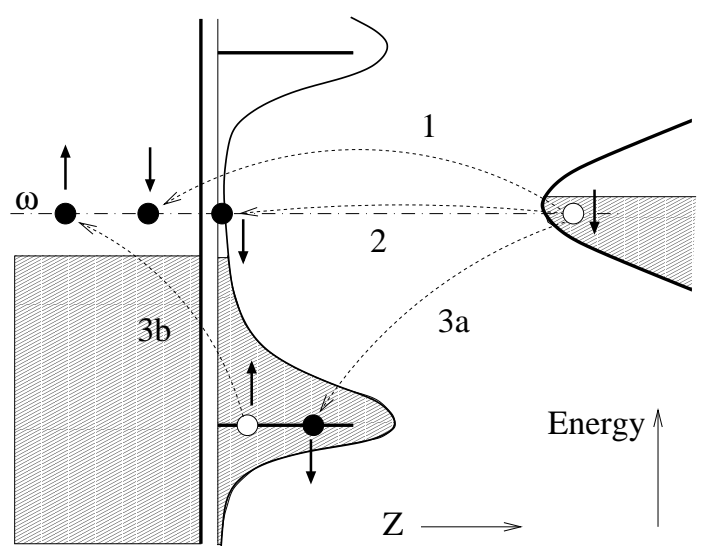

FIG. 8. Possible scattering channels for an electron tunneling from tip to metal through a magnetic impurity adsorbed on the surface.

We adopt the slave-boson technique of Coleman ${ }^{65}$ 
and find the adsorbate Green's function using the noncrossing approximation (NCA) ${ }^{13}$. Following the theory of section III, the final expression for current in Eq. (3.5) is valid, as well as all the consequent steps and approximation in III. We insert the solution for the Green's function $G_{a}$ of the $(U=\infty)$ interacting system in Eq. (3.27). This is equivalent to including the three tunneling channels in Fig. 8 to lowest order in the tip-system couplings. We now turn to the discussion of the results based on this approach.

\section{Results for large tip-substrate separation}

In order to model $C o / A u(111)$ studied both experimentally $^{21}$ and theoretically ${ }^{31}$, we choose a parameterization that gives the Kondo temperature $T_{K} \approx 70 \mathrm{~K}$ appropriate for the system. Our simplified model has degeneracy $N=2$ with no orbital degeneracy, band width $2 D=10 \mathrm{eV}$, and the adsorbate level at $\epsilon_{0}=0.75 \mathrm{eV}$ with the width $2 \Gamma=1 \mathrm{eV}$ (the width of a multiplet with an occupied level is $N \Gamma$ rather than $\Gamma$ !). We show the corresponding spectral function and the real part of $G_{a}$ in the inset of Fig. 9.
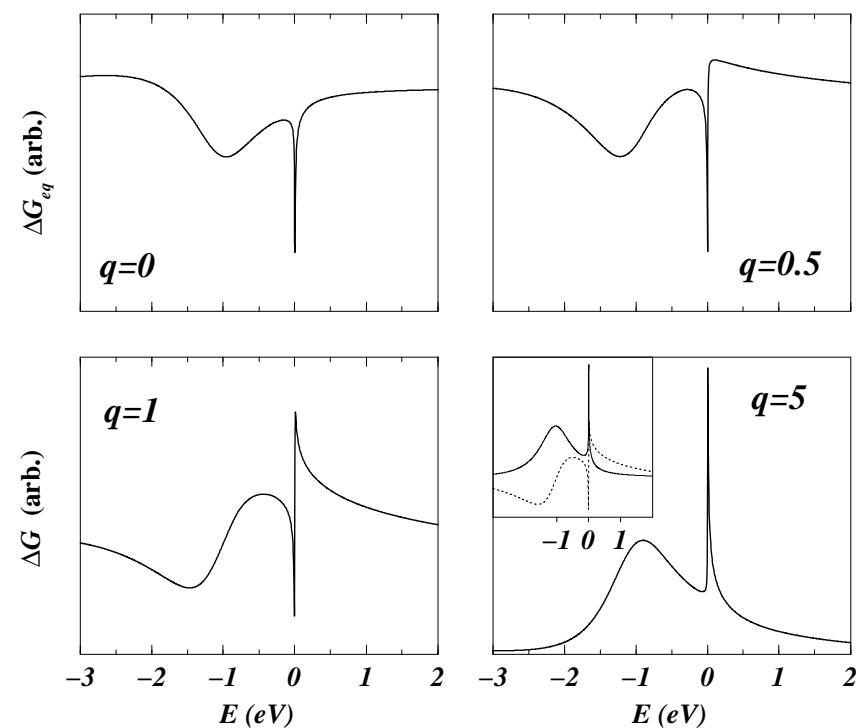

FIG. 9. The spectral lineshape for a model Kondo system described in the text over large bias range that includes tunneling into the broad resonance at $0.75 \mathrm{eV}$ below the Fermi level. Each panel corresponds to different $q\left(\epsilon_{F s}\right)$ at the Fermi level.

Fig. 9 shows the spectral properties of the system using $\Delta \mathcal{G}_{e q}$ over the whole energy range of the conduction band and for different values of $q$ at the Fermi level. The spectrum contains information about the broad resonance at $\epsilon_{0}=0.75 \mathrm{eV}$ below the Fermi level, as well as the prominent feature due to the Kondo resonance at zero bias. We show the large bias voltage results only for completeness since we do not expect the STM experiments to be able to provide spectroscopic information about the system over the whole energy range shown.

The resonance lineshapes both in $C o / A u(111)$ and $\mathrm{Ce} / \mathrm{Ag}(111)$ correspond to small values of $q$. Madhavan et $a l .{ }^{21}$ fitted the observed resonances to Fano lineshapes with $q \sim 0.7$. Our best fit would give approximately the same value of $q$. In the case of $C e / A g(111)$, the observed feature is an almost symmetric antiresonance corresponding to $q \sim 0$. Due to the contribution from the substrate electronic structure to $q$, its value cannot be directly used to make quantitative statements about the relative strength of the tunneling into the discrete state $d(f)$ with respect to that into the continuum. However, in agreement with $\mathrm{Li}$ et $a .^{22}$ and Lang ${ }^{66}$ we conclude that the STM probes mostly the $s p$ wave functions and the tunneling into the $f$-orbital is rather weak at the tipadsorbate distances used in the $C e / A g(111)$ experiment. The resonance is mostly the result of interference between conduction electrons scattering from the impurity. The larger value of $q$ in $C o / A u(111)$ indicates stronger contribution from the coupling of the STM to the $d$ orbital. This is expected because the $3 d$ orbital is not as tightly bound.

The recent work of Kawasaka et al. ${ }^{50,31}$ deals with the spatial and spectroscopic profiles of the Kondo resonance. They begin with the Tersoff-Hamann ${ }^{24}$ expression for current (3.12) and use the local density of states given by (3.14). They insert the self energy correction in the Green's function $G_{a}$ due to the intra-adsorbate Coulomb correlations using perturbation theory $\left(T>T_{K}\right)$ and Yamada's expansion in $U\left(T<T_{K}\right)$ to study the temperature dependence in the whole temperature range. They neglect the additional temperature effects due to the Fermi surface broadening, replace $(-\partial f / \partial \omega)$ by the delta function, and evaluate the conductance at the tip bias.

One of the main conclusions of their work ${ }^{31}$ is that the calculated temperature dependence of the resonance in the differential conductance is indicative of the temperature dependence of the Kondo resonance itself. They show results at the experimentally relevant low temperatures for $C o / A u(111)$ and $C e / A g(111)$ in the range of temperatures $\left(T \leq 0.1 T_{K}\right)$. They find a rather weak temperature dependence, due entirely to the temperature dependence of the spectral function $\rho_{a}$. It is qualitatively the same and comparable in magnitude with that found in Fig. 7 for a temperature independent resonance of the noninteracting system for temperatures $T \leq \Gamma$. In our case, the temperature dependence in $\Delta \mathcal{G}_{e q}$ is the consequence of the Fermi surface broadening in the STM tip. Therefore a careful deconvolution is necessary even at these low temperatures to extract information about the temperature dependence of the Kondo resonance. The other possibility is to eliminate variations in the Fermi surface broadening of the tip.

We show the temperature dependence for a Kondo system in Fig. 10. Since the validity of our approximation is limited to temperatures of order $T_{K}$ and higher, we 
show our results only in this temperature range. Panel (c) shows the temperature dependence one would observe with the tip at $T=0 \mathrm{~K}$ and with varying substrate temperature, i.e. when only the temperature dependence of the spectral function is taken into account. Panel (b) assumes the substrate is at a constant temperature $T=T_{K}$, which determines the shape of the Kondo resonance, while the tip temperature is varied. We see that the two contributions produce a very similar broadening of the Fano resonance. Only a close look can uncover the difference. Panel (a) shows the combined effect when the tip and substrate are kept at a common temperature. Obviously, it would be difficult to determine the contribution from the broadening of the Kondo resonance.

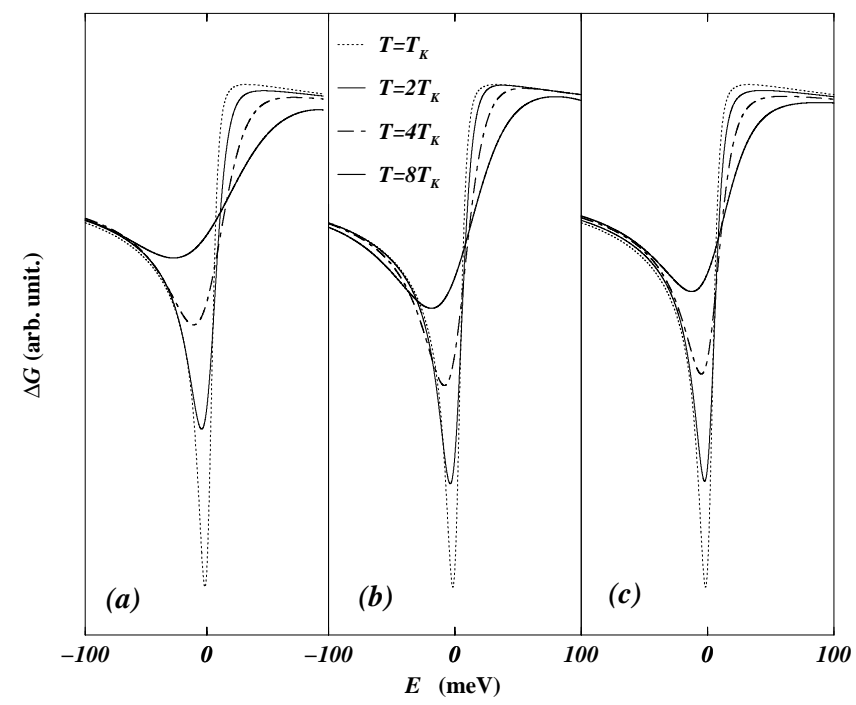

FIG. 10. Temperature dependence of the tunneling conductance through the Kondo resonance. (a) the total dependence includes the temperature dependence in the spectral function and the Fermi surface broadening; (b) temperature dependence due to the Fermi level broadening and with spectral function given by its $T \approx T_{K}$ value for all temperatures; (c) temperature dependence of only the spectral function without the Fermi surface broadening (replaced by delta function).

In addition to the temperature effects just discussed, Kawasaka et al. ${ }^{50,31}$ also predicted the existence of weak, long range oscillations in the current as a function of the lateral tip position. The particular long wavelength, long range character of these predicted oscillations are a consequence of their assumption that the tip-to-metal tunneling takes place into the surface states of the (111) noble metal surfaces. The observed resonances ${ }^{21,22}$ are at variance with these expectations. On the other hand, the limited spatial extent of the resonance observed at lateral tip positions up to $10 \AA$ is consistent with the rapid spatial decay determined by the bulk $G_{0}^{+}$. No significant changes in the resonance lineshape are expected on this length scale (see discussion in section III G and Fig. 6). The surface state would, however, be responsible for line- shapes variations on larger length scales of order $20 \AA$. The fact that no resonance is observed at such a distance from the impurity indicates that the surface state contribution is indeed weak. Since Friedel oscillations have been observed over long tip-impurity separations, we believe that a weak tunneling resonance most likely persists in the conductance over comparable distances, but more sensitive experiments are necessary. In this case, changes in the lineshape with $R_{\|}$are expected. However, unlike Schiller and Hershfield ${ }^{32}$, we do not expect variations in the lineshape due to the dominant contribution from the bulk states on the length scale of $\leq 5 \AA$ as discussed in section IV A 3.

\section{Nonequilibrium and hybridization effects at small tip-substrate separation}

In typical STM experiments, the tip-substrate separation can be varied from the point of contact where the tunneling resistance $R$ is a few $100 k \Omega$ to distances where $R \sim 1 G \Omega$. Experimental constraints limit the STM usefulness to the near Fermi level spectroscopy - especially at small $Z_{t}$ - because of exponentially increasing tunneling currents with bias. However, it is likely to be possible to investigate the Kondo resonance - which only requires biases of the order of $\sim 10 \mathrm{meV}$ - with very small tipadsorbate separations. It is therefore useful to analyze the physical consequences of the small tip-metal separation on the resonance in tunneling conductance.

In this case, nonequilibrium effects, as well as the tipadsorbate interaction, become important in the spectroscopy of Kondo systems. First of all, as $\Gamma_{a t}$ increases and becomes a significant fraction of $\Gamma_{a s}$ at small distances, the tip-adsorbate hybridization will contribute to the width $\Gamma$ of the resonance and to the renormalization of the level $\epsilon_{0}$. As a result, the Kondo temperature, which depends sensitively on $\Gamma$ and $\epsilon_{0}$, will change. This could be particularly important for systems with very low bulk $T_{K}$, such as $\mathrm{Fe} / \mathrm{Au}$ with $T_{K} \sim 1 \mathrm{~K}$. The Kondo temperature for an impurity adsorbed on the surface of the metal is even lower than its bulk $T_{K}$ because the lower coordination number for the adsorbate makes the width $\Gamma$ narrower. If $T_{K} \ll T$, the Kondo resonance will not be observed. In certain systems and in the right temperature regime, it may be possible for the Kondo resonance to reappear at smaller tip-adsorbate distance as a result of the increased hybridization. This could also be achieved by incorporating the adsorbate into the top surface layer. The recent study of transition-metal impurities at the surface of gold ${ }^{67}$ did not find any sign of the Kondo effect in $V, C r, M n$, or $F e$. We believe that, in the case of iron, this is due to the low $T_{K}$ and may be an example of a candidate system for the conditions discussed here. On the other hand, the smaller impuritymetal hybridization at the surface can lead to magnetic behavior for systems which are nonmagnetic in the bulk, 
such as $\mathrm{Ni} / \mathrm{Cu}$. There is a possibility for observing the transition between magnetic and nonmagnetic behavior on a single system induced either by embedding or by the proximity of the STM tip.

We show an example of the changing $T_{K}$ with hybridization in Fig. 11, where the spectral function $\rho_{a}(\omega)$ is plotted at zero bias as a function of the partial width $\Gamma_{a t}$, i.e. tip-metal separation for a model system. We choose $D=5 \mathrm{eV}, \Gamma_{a s}=0.25 \mathrm{eV}, \epsilon_{a}=-1 \mathrm{eV}$, and $T=30 \mathrm{~K}$. The Kondo temperature for this model in the limit $t_{a}=0$, is $T_{K} \sim 30 \mathrm{mK}$, much smaller than the temperature $T$. Therefore the Kondo resonance in the spectral function is very weak. When the tip is brought closer to the adsorbate, the Kondo resonance acquires more spectral weight as the Kondo temperature increases to $T_{K} \sim 100 \mathrm{mK}$ at $\Gamma_{a t}=0.01 \Gamma_{a s}, T_{K} \sim 0.2 \mathrm{~K}$ at $\Gamma_{a t}=0.04 \Gamma_{a s}$, and $T_{K} \sim 1.5 \mathrm{~K}$ at $\Gamma_{a t}=0.25 \Gamma_{a s}$. Based on the justifications in Appendix A, we neglected the effect of the direct metal-tip interaction on the spectral function and treat the effect of the tip as another hybridization channel for the impurity state.

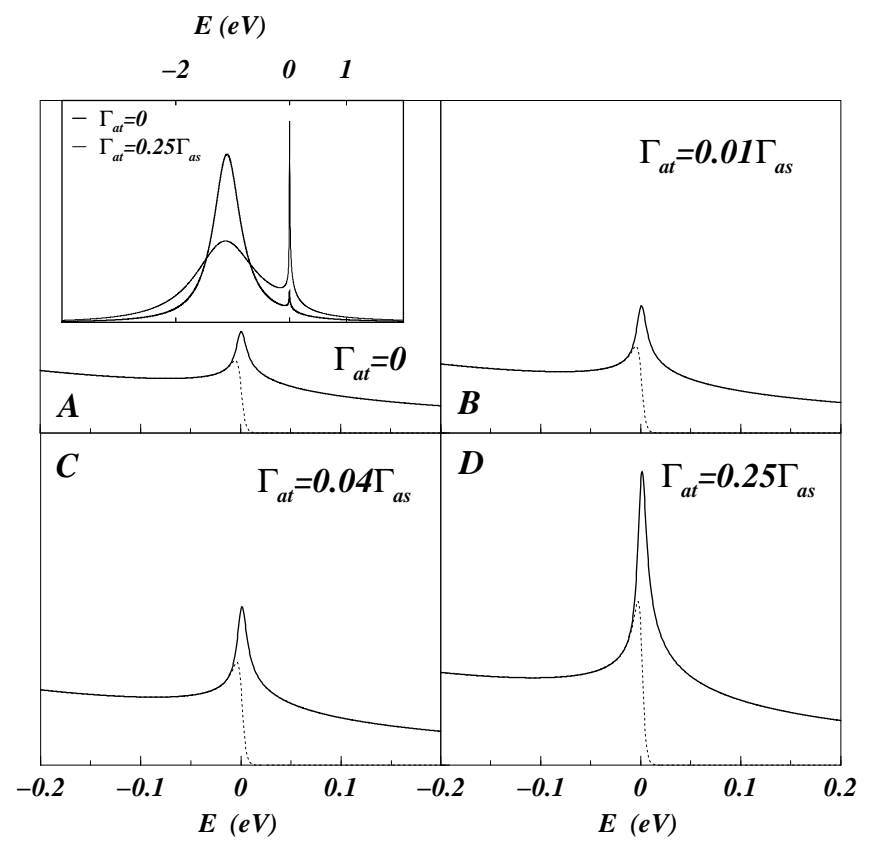

FIG. 11. The spectral function $\rho_{a}(\omega)$ at zero bias as a function of the tip-substrate separation (defined in terms of $\Gamma_{a t}$ ). Dotted line $=$ occupied density of states, bold line $=$ spectral density of states.

The effect of varying hybridization - due to either the presence of the STM tip or due to embedding or changing the environment of the adsorbate - on the tunneling resonance depends on the relation between $T_{K}$ and $T$. For instance, when $T_{K} \ll T$, increased hybridization would produce stronger (sharper) tunneling resonance of the same width since the spectral weight in the Kondo resonance increases while its width remains almost constant until $T_{K} \sim T$. Also the experimental resolution is limited by temperature in this regime. When, on the other hand $T_{K} \geq T$, additional hybridization would not only increase the spectral weight in the Kondo resonance but also its width. The two cases should thus be distinguishable experimentally from each other and from the possible lineshape variations with $Z_{t}$ as a result of changing $q$.

The second important effect of the strong tip-adsorbate interaction is the breakdown of equilibrium relations at finite bias such as the fluctuation-dissipation theorem $G^{<}(\omega)=f(\omega) \rho_{a}(\omega)$ which consequently cannot be used in deriving the expression for current (3.24). This is true in general because the electron occupation of the tip, metal, and adsorbate electrons will no longer be thermal, i.e. will not be given by $f_{t}(\omega)$ and $f_{s}(\omega)$ but rather will be characterized by a nonequilibrium distribution produced by the injected tunnel electrons. The differential conductance is no longer proportional to the local density of states and cannot be obtained using Eq. (3.27). In Kondo systems, the hot electrons not only modify the electronic distribution on the impurity, but also modify the Kondo resonance itself.

This is shown Fig. 2 where the spectral function and density of occupied states is plotted at selected bias voltages in the limit of $\left|t_{p k}\right| \ll\left|t_{p a}\right|$. The impurity has a resonance at $\epsilon_{0}=-1 \mathrm{eV}$ below the Fermi level and total width $\Gamma=0.5 \mathrm{eV}$ produced by the hybridization with both the tip and the substrate with the partial widths $\Gamma_{a t}=0.1 \Gamma_{a s}$. Temperature is of the order of $T_{K}$ in this example. We see that the Kondo resonance broadens even more with increasing bias. This is due to the increase in the rate of incoherent scattering by $\sim e V_{a} / T$ - an effect similar to temperature. At the same time, the electron occupation develops a non-thermal profile due to the large tip-adsorbate current. This is particularly visible for negative biases where the density of states is larger. Fig. 2(a) shows the equilibrium spectral function (dotted) and the electron population on the resonance (solid bold). The equilibrium spectral density is shown (dotted) in all panels. In addition, the spectral density (solid) and occupation (bold solid) are shown for the biases indicated in the figure by the labeled arrow. If the coupling to the tip were comparable with the metal-adsorbate hybridization, a double peak structure would develop. This has been predicted by Wingreen and Meir ${ }^{51,52}$ in the context of the nonequilibrium Kondo effect in quantum dots, also discussed by Plihal et al. ${ }^{68}$. We see the onset of the double peak structure in panels (b)-(d) where a small cusp develops at the chemical potential of the tip. In summary, the bias has a significant effect on the spectral density even when $\Gamma_{a t} \sim 0.1 \Gamma_{a s}$.

We show the tunneling current (right) and the corresponding differential conductance (left) in Fig. 12 for this model of Kondo impurity and for the STM geometry defined by $\Gamma_{a t}=0.1 \Gamma_{a s}$ and $\left|t_{p k}\right| \ll\left|t_{p a}\right|$. The panels correspond to $q=0.6, q=1.2$, and $q=2.4$, respectively. The current on the right is calculated using $I_{t o t}=I_{e q}+\delta I_{n o n}$ of section IIID with $\delta I_{n o n}$ given by 
Eq. (3.26). The differential conductance $\mathcal{G}_{\text {tot }}$ on the left is obtained by differentiating the results displayed on the right. It cannot be calculated from the expressions in the text since the dependence of $\rho_{a}(\omega)$ and $G_{a}^{<}(\omega)$ on the bias voltage modifies the contributions to the current in a wide energy range, and $\mathcal{G}_{t o t}$ is not related in simple terms to the properties at the Fermi level of the tip. We compare $I_{t o t}$ and $\mathcal{G}_{t o t}$ (circles) with $I_{e q}$ and $\mathcal{G}_{e q}$ (solid). The equilibrium quantities were obtained in the lowest order in $t_{a p}$ and $t_{k p}$ and with the equilibrium $G_{a}$ of Fig. 2(a).

We see that the broadening and disappearance of the Kondo resonance with increasing bias at strong tipadsorbate coupling is weakened in the nonequilibrium calculation of $\mathcal{G}_{\text {tot }}$, because the contribution $\delta I_{\text {non }}$ compensates partially for the spectral function effect. The most consistent effect on the lineshape for various values of $q$ is the suppression of the resonance maximum and as a consequence a more symmetric appearance. This behavior is qualitatively different from both the hybridization effect and that of the changing Fano parameter $q$ - due to different decay constant of the impurity and metal states. Although the dependence of the tunneling resonance on the tip-substrate separation $Z_{t}$ will contain all three contributions, the hybridization and nonequilibrium contribution should only be important at extremely small tip-adsorbate separations. The variations in $q$ should not be important as it depends on the difference of the wavefunction tails and the two remaining contributions should leave distinguishable signatures in the tunneling resonance. It remains to be seen if the nonequilibrium condition play an important role in the tunneling between STM tip and Kondo impurity.

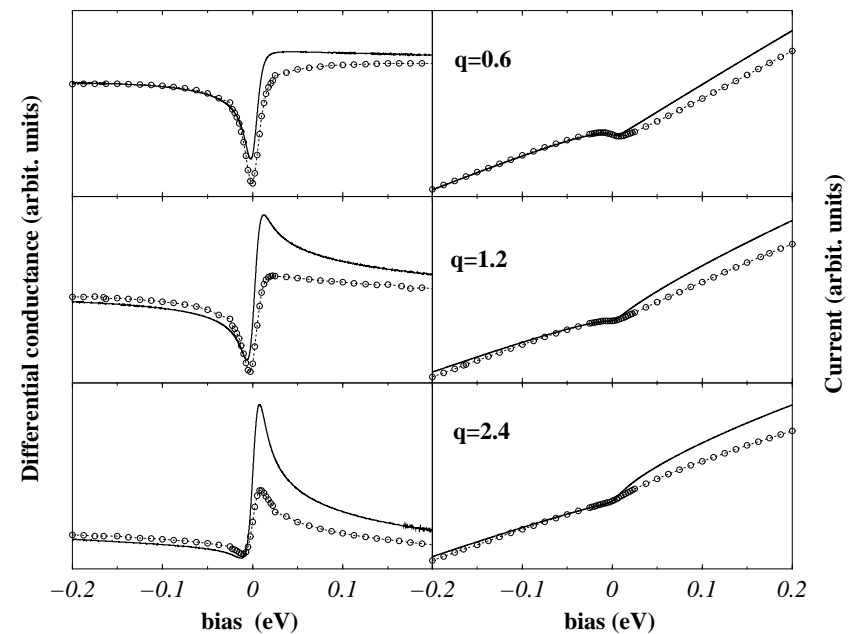

FIG. 12. Differential conductance (left) and current (right) for a Kondo system defined in the text and with the spectral function displayed in Fig. 2. Solid line is the calculation in the lowest order in $t_{k p}$ and $t_{a p}$ and with the equilibrium spectral function of Fig. 2. Circles correspond to the nonequilibrium $\mathcal{G}_{t o t}=d I_{t o t} / d V$ and $I_{t o t}$ for $\Gamma_{a t}=0.1 \Gamma_{a s}$. Each row corresponds to a given value of $q$ at the Fermi level.
Finally, we note that the limit $\left|t_{p k}\right| \ll\left|t_{p a}\right|$ discussed here in connection with the nonequilibrium effects is not appropriate for the recent STM experiments, where $t_{p a}$ is likely much weaker than $t_{p k}$, even though the importance of $t_{p a}$ will increase relative to $t_{p k}$ with decreasing $Z_{t}$. We will address the more general case in a future work.

\section{CONCLUSIONS}

We used the Keldysh-Kadanoff method to study the spectroscopic features of adsorbate resonances in the STM tunneling experiments. The central results of our theory are the general expression for current Eq. (3.5) to all orders in the tunneling matrix elements and its equilibrium limit (3.11). Both are valid for arbitrary intra-adsorbate electron correlations and thus apply to both noninteracting $(U=0)$, as well as magnetic (large $U)$ systems. The discussion of the Fano resonances is based on additional approximations for the tunneling and hybridization matrix elements that lead to expressions (3.24) for the tunneling current and Eq. (3.27) for differential conductance in the lowest order in the tip-tosystem tunneling matrix elements $t_{a}$ and $t_{c}$, i.e. at large tip-surface separation, and the nonequilibrium correction to the current in Eq. (3.26).

In the equilibrium limit, our theory of the tunneling current and conductance differs from the standard theories of STM, in that the dependence on LDOS is replaced by a tip specific quantity related to the LDOS (Eq. 3.9). The current is expressed entirely in terms of the adsorbate Green's function $G_{a}^{R}$, the tip density of states, the tunneling matrix elements and the substrate Green's function. We used the formulation to study the resonance lineshape as a function of temperature, tipsubstrate separation, and lateral tip position. We summarize our findings as follows.

(1) The role of impurity state resonances in tunneling can be discussed in terms of two limiting cases. When direct tunneling across the barrier is weak, the resonance within the barrier provides an additional tunneling channel and can significantly enhance the tunneling current. This is the case of quantum dots in Coulomb blockade regime. If on the other hand, the tunneling into the continuum is strong, the presence of an "impurity" state could suppress the tunneling current due to the additional scattering of the conduction electrons in the metal from the impurity, i.e. increased resistance. The tunneling into the Kondo resonance in the recent STM experiments seems to be closer to the latter limit.

(2) The information about electron correlations and the Kondo resonance enters the tunneling problem through the impurity Green's function $G_{a}$ while the position dependence of the conductance is controlled by the electronic structure of the metal.

(3) The spatial decay of the observed Fano resonance in the recent experiments ${ }^{21,22}$ is consistent with the con- 
clusion that tunneling into the bulk conduction and hybridized $s p$ impurity states gives rise to most of the signal. The absence of any observable resonance at distances larger than $\sim 10 \AA$ suggests that the contribution from the surface state on $\mathrm{Au}(111)$ and $\mathrm{Ag}(111)$ to the resonant tunneling is not important in these experiments. However, the surface states are important in special cases, as indicated by the recent corral experiments ${ }^{64}$ in which the contribution of the surface states is enhanced by scattering from the walls of the corral.

(4) At large $Z_{t}$, tunneling into conduction states with $k_{\|}$having the smallest parallel component corresponding to energy $\omega=\epsilon_{k_{\perp}}+\epsilon_{k_{\|}}$is strongly favored. This leads to the disappearance of the current oscillation vs. the lateral tip position due to tunneling into the bulk states which should otherwise be observed with period of about $1-2 \AA$ (corresponding to the bulk $k_{F}$ ) for typical experimental conditions. Therefore no oscillations in the lineshape should be observed on this length scale for typical tip-surface separation. The occurrence of an antiresonance with tip position at certain neighboring sites predicted by Schiller and Hershfield ${ }^{32}$ has its origin in these oscillations. It is a result of a simplified model for the surface electronic structure and we believe is unphysical. The small current oscillations predicted by Kawasaka et $a l .{ }^{31}$ assume that the surface states are all-important in the spatial dependence of the resonance which seems to contradict the experimental results. We believe the surface state should be important at larger distances since on the (111) noble metal surfaces $k_{F} \sim 0.15-0.2 \AA^{-1}$ and the corresponding period of oscillations is about $20 \AA$ (as observed experimentally as Friedel oscillations). We expect changes in the resonance lineshapes with this spatial period if the contribution from the surface state if the signature of the resonance is detectable at such distances.

(5) From the lineshapes observed in $C o / A u(111)$ and $\mathrm{Ce} / \mathrm{Ag}(111)$, we conclude that the direct tunneling into the discrete $(d$ or $f$ ) state is quite weak - stronger in $C o / A u(111)$. This confirms that the STM is mostly a probe of the delocalized $s p$ states and couples only weakly to the tightly bound $d$ or $f$ orbitals at typical tip-surface separations. Therefore the dominant process giving rise to the resonance lineshape is the tip-to-metal tunneling and interference between conduction electrons scattering from the local moment.

(6) The temperature dependence in differential conductance does not reflect only the temperature dependence of the Kondo resonance, but includes also the effect of Fermi surface broadening (mostly of the tip). The two contributions are of the same order of magnitude and qualitatively indistinguishable. Therefore, the temperature dependence in the differential conductance cannot be used directly to make conclusions about the temperature dependence of the resonance without controlling the tip Fermi surface broadening or without deconvolution.

(7) At small tip-surface separations, nonequilibrium effects as well as the additional tip-adsorbate hybridization may play an important role - especially in Kondo systems. The main effect of the finite bias voltage in this case is to broaden the Kondo resonance and produce nonequilibrium electron population on the adsorbate. The observed Fano resonance in differential conductance also broadens and its maximum is suppressed. The effect of the tunneling current on the Kondo resonance should thus leave a characteristic dependence of the lineshape on $Z_{t}$.

\section{ACKNOWLEDGMENTS}

We thank R. Celotta, E. Hudson, M. Stiles, and J. Stroscio for fruitful discussions and for helping us understand the experimental issues more clearly.

\section{Appendix A. ADSORBATE GREEN'S FUNCTION}

An important quantity in the theory of tunneling current through adsorbate resonances is the adsorbate Green's function $G_{a}$. Using the equation of motion method, we find the expression for $G_{a}$ defined as the Fourier transform of

$$
G_{a}\left(t, t^{\prime}\right)=-i\left\langle T_{C} c_{a}(t) c_{a}^{\dagger}\left(t^{\prime}\right)\right\rangle .
$$

We do this for the case of arbitrarily strong coupling between the tip and the adsorbate with the intent to describe the nonequilibrium effects at finite bias. However, in this paper we consider the effect of the direct tip-metal interaction on $G_{a}$ to be weak and neglect it. Extension to the full description will be considered in future work. We believe the approximations adopted here capture the most important nonequilibrium effects.

We discuss both the noninteracting $(U=0)$ and interacting $(U=\infty)$ model. Since the solution in both limits for the adsorbate-metal interaction is well known, we limit our discussion to the issues specific to the addition of the biased tip and refer reader to standard texts for the details. The $(U=\infty)$ model is solved using the slave boson technique and NCA. In this approach a new pseudofermion is introduced by the transformation $c_{a} \rightarrow c_{a} b^{\dagger}$ in the Hamiltonian (2.1), where $b^{\dagger}$ is the creation operator for the slave boson. This eliminates the interaction term $U$ from the Hamiltonian as discussed by Coleman. ${ }^{65}$

The time ordering operator $T_{C}$ orders the time according to their position on contour in the complex time plane $^{47}$. It is important to note that the equations must be first solved in the complex time domain and then analytically continued to the real axis as was pointed out in the previous appendix. The analytic continuation is performed before the Fourier transform, so we must be careful about how we deal with the Fourier transformed equations. Relevant details are in Appendix C. Here we discuss the equations of motion satisfied by the Fourier transforms of the time ordered Green's functions and the analytic continuation is performed at the end according 
to the rules in Appendix C. All Green's functions and self-energies in the following expressions are function of frequency $\omega$ and, therefore, we omit their argument to simplify the notation.
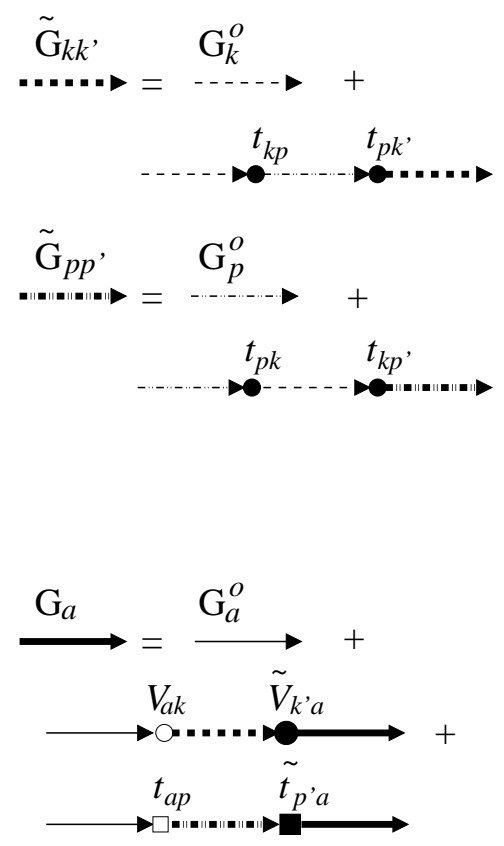

FIG. 13. (a) Diagrammatic expansion for the metal $\left(\tilde{G}_{k k^{\prime}}\right)$ and tip $\left(\tilde{G}_{p p^{\prime}}\right)$ Green's functions in mutual interaction but without the adsorbate. (b) diagrammatic expansion for the adsorbate Green's function $G_{a}$ of the noninteracting Anderson model using the solutions $\tilde{G}_{k k^{\prime}}$ and $\tilde{G}_{p p^{\prime}}$.

The Green's function for the impurity state $G_{a}$ can be written in a standard way

$$
G_{a}=\left(\omega-\epsilon_{0}-\Sigma_{a}\right)^{-1},
$$

using the self energy $\Sigma_{a}\left(\vec{R}_{t}, Z_{0} ; \omega\right)$. The solution for $G_{a}$ is thus reduced to finding $\Sigma_{a}$. We first treat a closed shell or nonmagnetic open shell $\left(V_{k a} \gg U\right)$ adsorbate for which electron correlations can be neglected. We begin by considering the tip-substrate system without the adsorbate. We define $\tilde{G}_{k k^{\prime}}$ and $\tilde{G}_{p p^{\prime}}$ in analogy with $G_{a}(\mathrm{~A} 1)$ as the Green's functions of the metal and tip states, respectively, in the absence of the adsorbate. These are not identical with the Green's functions $G_{k k^{\prime}}$ and $G_{p p^{\prime}}$ for the full system introduced in Appendix B. The bare metal-tip system is described by the Hamiltonian of section II with $\epsilon_{0}=U=t_{a p}=V_{a k} \equiv 0$. Using the equations of motion, we can write

$$
\left(\omega-\epsilon_{k}\right) \tilde{G}_{k k^{\prime}}=\delta_{k k^{\prime}}+\sum_{k^{\prime \prime}} \Sigma_{k k^{\prime \prime}} \tilde{G}_{k^{\prime \prime} k^{\prime}}
$$

and

$$
\left(\omega-\epsilon_{p}\right) \tilde{G}_{p p^{\prime}}=\delta_{p p^{\prime}}+\sum_{p^{\prime \prime}} \Sigma_{p p^{\prime \prime}} \tilde{G}_{p^{\prime \prime} p^{\prime}}
$$

where the self-energies are $\Sigma_{k k^{\prime}}=\sum_{p} t_{k p} G_{p}^{0} t_{p k^{\prime}}$ and $\Sigma_{p p^{\prime}}=\sum_{k} t_{p k} G_{k}^{0} t_{k p^{\prime}}$ and $G_{k}^{0}=\left(\omega-\epsilon_{k}+i \eta_{k}\right)^{-1}$ and $G_{p}^{0}=\left(\omega-\epsilon_{p}+i \eta_{p}\right)^{-1}$ are the Green's functions for the clean metal and tip, respectively, without their mutual interaction. The coupled equations are shown diagrammatically in Fig. 13. The solutions for $\tilde{G}_{k k^{\prime}}$ and $\tilde{G}_{p p^{\prime}}$ can be formally written as the inverse of $D_{k k^{\prime}}=$ $\delta_{k k^{\prime}}\left(\omega-\epsilon_{k}\right)-\Sigma_{k k^{\prime}}$ and $D_{p p^{\prime}}=\delta_{p p^{\prime}}\left(\omega-\epsilon_{p}\right)-\Sigma_{p p^{\prime}}$. We also define the adsorbate-metal and adsorbate-tip hybridization matrices modified by the tip-substrate interaction as $\tilde{V}_{k a}=V_{k a}+\sum_{p} t_{k p} G_{p}^{0} t_{p a}$ and $\tilde{t}_{p a}=t_{p a}+\sum_{k} t_{p k} G_{k}^{0} V_{k a}$. With these definitions and with $\tilde{G}_{k k^{\prime}}$ and $\tilde{G}_{p p^{\prime}}$ obtained through (A3) and (A4), the solution for the noninteracting $\Sigma_{a}$ - shown diagrammatically in Fig. 13 - is formally given by

$$
\Sigma_{a}=\sum_{k k^{\prime}} V_{a k} \tilde{G}_{k k^{\prime}} \tilde{V}_{k^{\prime} a}+\sum_{p p^{\prime}} t_{a p} \tilde{G}_{p p^{\prime}} \tilde{t}_{p^{\prime} a} .
$$

The evaluation of the self energy $\Sigma_{a}$ is rather complicated in the general case of strong tip-to-substrate coupling. We proceed with formulation of the general nonequilibrium theory for the tunneling current using this self energy (section III A) and then we discuss two limiting cases: (a) the equilibrium limit $\left|t_{k p}\right|,\left|t_{a p}\right| \ll\left|V_{k a}\right|$ (section IIIC) in which case the second term in (A5) is neglected and $\tilde{V}_{k a}, \tilde{G}_{k k^{\prime}}$ replaced by $V_{k a}, G_{k}^{0} \delta_{k k^{\prime}}$; and (b) the nonequilibrium case under the assumption $\left|t_{k p}\right| \ll\left|t_{a p}\right| \sim\left|V_{k a}\right|$, in which case we keep both terms in (A5) and replace $\tilde{V}_{k a}, \tilde{t}_{a p}, \tilde{G}_{k k^{\prime}}$, and $\tilde{G}_{p p^{\prime}}$ by $V_{k a}, t_{a p}$, $G_{k}^{0} \delta_{k k^{\prime}}$, and $G_{p}^{0} \delta_{p p^{\prime}}$. Section IV B 3 deals with tunneling through a Kondo impurity in this limit. The case (b) includes the effect of the increased hybridization of the discrete state due to the tip presence and the onset of nonequilibrium population on the adsorbate at finite bias.

In order to study these corrections in the limit (b), $\left(\left|t_{p k}\right| \ll\left|t_{p a}\right| \sim\left|V_{k a}\right|\right)$, we replace the Green's functions $\tilde{G}_{k k^{\prime}}$ and $\tilde{G}_{p p^{\prime}}$ by the noninteracting ones, i.e. $\tilde{G}_{k k^{\prime}}=$ $\delta_{k k^{\prime}} G_{k}^{0}$ and $\tilde{G}_{p p^{\prime}}=\delta_{p p^{\prime}} G_{p}^{0}$ and the modified $\tilde{V}_{k a}$ and $\tilde{t}_{p a}$ by $V_{k a}$ and $t_{p a}$. The self energy $\Sigma_{a}$ then simplifies to

$$
\Sigma_{a}^{0}=\sum_{k}\left|V_{a k}\right|^{2} G_{k}^{0}+\sum_{p}\left|t_{a p}\right|^{2} G_{p}^{0}
$$

The largest source of error in writing the approximate self energy is the neglect of the possibly significant interference effects at larger $t_{k p}$ as a result of the phase difference between $\tilde{t}_{p a}$ and $t_{p a}$. It is always reasonable to replace $\tilde{V}_{k a}$ by $V_{k a}$, as long as the adsorbate is on the surface rather than on the STM tip. These general case will be the topic of a future study. If the tip distance from the adsorbate is much larger than the adsorbatemetal separation, so that $t_{a p} \ll V_{a k}$, the self-energy is well described by the first term only. In such a case, the STM does not strongly modify the studied system. It is then reasonable to characterize the system without the presence of the STM tip and then consider the tunneling. 
Finally, we discuss the Green's function $G_{a}$ in the limit (b) for a Kondo impurity which is likely to show stronger dependence on the bias and tip interaction. We find the nonequilibrium Green's function $G_{a}$ under the same assumption that lead to $\Sigma_{a}^{0}$ for the noninteracting Anderson Hamiltonian. We solve the interacting system in the limit of $U=\infty$ using the NCA approximation, shown diagrammatically in Fig. 14. The self energy is not a simple sum of the two contributions from the metal and tip as it was in the noninteracting system, because the occupation of the resonance is limited to one electron and the hybridization is now correlated - formally through the slave boson Green's function $B(\omega)$. The two coupled equations in Fig. 14 are solved selfconsistently. The expressions for the NCA self energy is obtained in a standard with the help of the diagrams in Fig. $14 .^{13}$
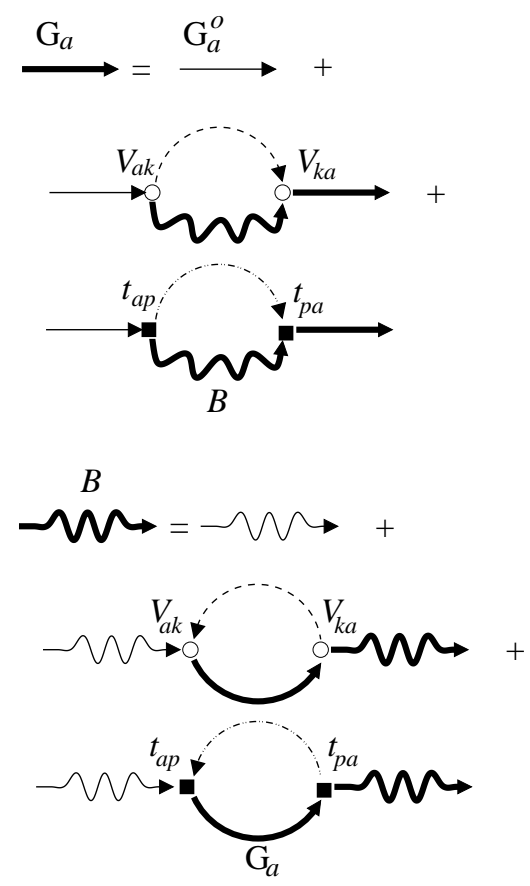

FIG. 14. The diagrammatic expansion for the adsorbate Green's function $G_{a}$ of the interacting Anderson Hamiltonian $(U=\infty)$ within the NCA.

\section{Appendix B. EQUATIONS OF MOTION FOR $G_{P K}$ AND $G_{P A}$}

In this appendix, we find the solution for $\left(\sum_{k p} t_{k p} G_{p k}+\sum_{a p} t_{a p} G_{p a}\right)$ entering the expression for tunneling current (3.3) for the general case of arbitrary tip-system coupling. Ultimately, the interesting regime in connection with typical STM experiments is one in which $t_{k p}, t_{a p}<V_{a k}$. However, we want to be able, in principle, to study the system when the coupling of the STM tip to the system and the tunneling current are strong. This creates nonequilibrium occupation on the adsorbate res- onance and modifies the spectroscopic properties of the system. We therefore proceed by deriving the most general expression valid for arbitrary coupling strength $t_{k p}$ and $t_{a p}$ and discuss an approximation (b) that allows us to take into account the most important nonequilibrium effects as described in the previous appendix. For this purpose we introduce Green's function $G_{p a}(\omega), G_{p k}(\omega)$, $G_{k k^{\prime}}(\omega), G_{p p^{\prime}}(\omega)$, and $G_{k a}(\omega)$ as the Fourier transform of

$$
G_{i j}\left(t, t^{\prime}\right)=-i\left\langle T_{C} c_{i}(t) c_{j}^{\dagger}\left(t^{\prime}\right)\right\rangle
$$

We now turn to the equations of motion for the Green's functions relevant for the tunneling current. The following expressions are valid for arbitrary interaction $U \neq 0$ and the nature of the intra-adsorbate interactions are contained fully in the solution for $G_{a}$ discussed in the previous appendix. The first term in the current (3.3) contains the tip-adsorbate propagator which satisfies

$$
\left(\omega-\epsilon_{p}\right) G_{p a}=t_{p a} G_{a}+\sum_{k} t_{p k} G_{k a} .
$$

It is expressed in term of $G_{a}$ already solved within a given approximation in the previous appendix through (A2) and in terms of the metal-adsorbate Green's function

$$
\left(\omega-\epsilon_{k}\right) G_{k a}=V_{k a} G_{a}+\sum_{p} t_{k p} G_{p a} .
$$

The last two equations are coupled and need to be solved self-consistently. We do this by substituting Eq. (B3) for $G_{k a}$ in (B2), and vice versa. The solutions are then expressed in terms of $G_{a}, \tilde{G}_{p p^{\prime}}$, and $\tilde{t}_{p a}$ discussed in the previous appendix as

$$
G_{p a}=\sum_{p^{\prime}} \tilde{G}_{p p^{\prime}} \tilde{t}_{p^{\prime} a} G_{a} .
$$

We will also need the solution for $G_{a k}$. The tip-induced correction to $V_{k a}$ contributes to the phase of $V_{k a}$, as well as its magnitude, and could thus affect the lineshape significantly in the strong coupling limit. But it should be particularly weak when $t_{k p}, t_{p a} \ll V_{k a}$ and it will be safe to ignore it. We write

$$
G_{a k}=G_{a} \sum_{k^{\prime}} \tilde{V}_{a k^{\prime}} \tilde{G}_{k^{\prime} k}
$$

The second term in (B3) is negligible when the tipadsorbate separation is much larger than the adsorbatemetal separation. Neglecting this term is equivalent to replacing $\tilde{G}_{p p^{\prime}} \rightarrow G_{p}^{0} \delta_{p p^{\prime}}$ in (B4) and $\tilde{G}_{k k^{\prime}} \rightarrow G_{k}^{0} \delta_{k k^{\prime}}$, $\tilde{V}_{k a} \rightarrow V_{k a}$ in (B5). The tip-adsorbate Green's function $G_{p a}$ is then expressed entirely in terms of $G_{a}$ and the unperturbed conduction electron Green's functions.

The second term in (3.3) contains the tip-metal propagator $G_{p k}$, which satisfies 


$$
\left(\omega-\epsilon_{p}\right) G_{p k}=\sum_{a} t_{p a} G_{a k}+\sum_{k^{\prime}} t_{p k^{\prime}} G_{k^{\prime} k} .
$$

It is expressed in terms of $G_{a k}$ (B5) discussed in the previous paragraph and in terms of $G_{k^{\prime} k}$, the Green's function for the substrate conduction electrons

$$
\left(\omega-\epsilon_{k^{\prime}}\right) G_{k^{\prime} k}=\delta_{k k^{\prime}}+\sum_{a} V_{k^{\prime} a} G_{a k}+\sum_{p} t_{k^{\prime} p} G_{p k} .
$$

We see that $G_{k k^{\prime}}$ couples to $G_{p k}$, (B6), and also to $G_{a k}$, (B5), already solved in terms of $G_{a}$ and $\tilde{G}_{k k^{\prime}}$. The last two equations can be solved self-consistently to give

$$
G_{k^{\prime} k}=\tilde{G}_{k^{\prime} k}+\sum_{k_{1} k_{2}} \tilde{G}_{k^{\prime} k_{1}} \tilde{V}_{a k_{1}} G_{a} \tilde{V}_{k_{2} a} \tilde{G}_{k_{2} k} .
$$

and

$$
G_{p k}=\sum_{p^{\prime}} \tilde{G}_{p p^{\prime}}\left(t_{p^{\prime} k}+\sum_{a k^{\prime}} \tilde{t}_{p^{\prime} a} G_{a} \tilde{V}_{a k_{1}}\right) G_{k}^{0} .
$$

For the purpose of analytic continuation, it is important to keep track of the order in which the Green's functions appear in the product in the above equations. The "lesser" Green's functions are then obtained according to rules stated in Appendix C. The equilibrium limit of the theory is achieved by neglecting the last term in (B7) along with the equivalent approximations for $G_{a}$ and $G_{a k}$ discussed above. This removes the self-consistency requirement and neglects the effect of the tip on the substrate conduction electrons, but not on the tunneling current. The solution for $G_{k k^{\prime}}$ is then identical to that of the system without the tip.

\section{Appendix C. RULES FOR DEALING WITH NONEQUILIBRIUM GREEN'S FUNCTIONS IN FREQUENCY SPACE}

In this appendix we review the process of analytic continuation of the complex time contour expression to integrals on the real time axis. We follow Langreth's generalization $^{47}$ of Kadanoff-Baym's method ${ }^{46}$ described in detail by Haug and Jauho ${ }^{48}$. Four Green's functions appear in the nonequilibrium theory "lesser" $G^{<}$, "greater" $G^{>}$, retarded $G^{R}$, and advanced $G^{A 46-48,26}$. We frequently need to find the retarded (advanced) and "lesser" Green's function corresponding to Green's function $A$ time ordered in the complex time plane expressed as a product of $N$ time ordered functions $B \ldots Z$, i.e.

$$
A\left(t, t^{\prime}\right)=\int_{C} d \tau_{1} \ldots d \tau_{2} B\left(t, \tau_{1}\right) . . Z\left(\tau_{2}, t^{\prime}\right)
$$

where all functions are assumed fermion-like. The desired expressions analytically continued onto the real time axis $\operatorname{are}^{47}$

$$
A^{R(A)}\left(t, t^{\prime}\right)=\int_{-\infty}^{\infty} d \tau_{1} \ldots d \tau_{2} B^{R(A)}\left(t, \tau_{1}\right) \ldots Z^{R(A)}\left(\tau_{2}, t^{\prime}\right)
$$

which consists of only one term and

$$
\begin{aligned}
& A^{\gtrless}\left(t, t^{\prime}\right)=\int_{-\infty}^{\infty} d \tau_{1} \ldots d \tau_{3} \ldots d \tau_{2} \\
& {\left[\ldots+B^{R}\left(t, \tau_{1}\right) \ldots C^{\gtrless}\left(t, \tau_{3}\right) \ldots Z^{A}\left(\tau_{2}, t^{\prime}\right)+\ldots\right]}
\end{aligned}
$$

where each of the $N$ terms in the integral has exactly one function of the type $f \gtrless$, all functions to the left (right) of it are retarded (advanced), and each of the terms has the $f \gtrless$ in a different position.

We are dealing here with the case of time independent perturbations (steady state current). The double time propagators then only depend on the time difference $(t-$ $\left.t^{\prime}\right)$. The equations $(\mathrm{C} 2),(\mathrm{C} 3)$ can be Fourier transformed and we can write the time ordered Green's function $A(\omega)$ as a simple product

$$
A(\omega)=B(\omega) \ldots Z(\omega) .
$$

The rules for writing the expression for the "lesser" and retarded function in the frequency space are then directly carried out by Fourier transforming the equations (C2) and (C3). Leaving out the frequency arguments, we write

$$
A^{R(A)}=B^{R(A)} \ldots Z^{R(A)}
$$

and

$$
A^{\gtrless}=\ldots+B^{R} \ldots C^{\gtrless} \ldots Z^{A}+\ldots
$$

We note that it is important to keep track of the order in which the function $B \ldots Z$ appear in the time integral when the Fourier product is formed.

${ }^{1}$ Tunneling Phenomena in Solids, edited by E. Burstein and S. Lundqvist (Plenum, New York, 1969).

${ }^{2}$ C. B. Duke, Tunneling in Solids (Academic, New York, 1969).

${ }^{3}$ E. L. Wolf, Principles of Electron Tunneling Spectroscopy (Oxford University Press, New York, 1985).

${ }^{4}$ L. Esaki, I. Giaver, and B. D. Josephson, in Nobel Lectures in Physics 1971-1980, edited by S. Lundqvist (World Scientific, Singapore, 1992), pp. 109-164.

${ }^{5}$ R. Young, J. Ward, and F. Scire, Rev. Sci. Instr. 43, 999 (1972).

${ }^{6}$ G. Binnig and H. Rohrer, Rev. Mod. Phys 59, 615 (1987).

${ }^{7}$ Scanning Tunneling Microscopy, Vol.27 in Methods of Experimental Physics, edited by J. A. Stroscio and W. J. Kaiser (Academic, San Diego, 1993). 
${ }^{8}$ Scanning Tunneling Microscopy III: Theory of STM and Related Scanning Probe Methods, edited by R. Wiesendanger and H.-J. Güntherodt (Springer-Verlag, Berlin, 1993).

${ }^{9}$ R. Wiesendanger, Scanning Probe Microscopy and Spectroscopy (Cambridge Univ. Press, Cambridge, UK, 1994).

${ }^{10}$ C. J. Chen, Introduction to Scanning Tunneling Microscopy (Springer, Berlin, Heidelberg, New York, 1996).

11 J. Kondo, Prog. Theor. Phys. 32, 37 (1964).

12 J. Kondo, Solid State Physics 23, 183 (1969).

13 A. C. Hewson, The Kondo Problem to Heavy Fermions (Cambridge University Press, Cambridge, 1993).

14 J. A. Appelbaum, J. C. Phillips, and G. Tzouras, Phys. Rev 160, 554 (1967).

${ }^{15}$ C. B. Duke and M. E. Alferieff, J. Chem. Phys. 46, 923 (1967).

${ }^{16}$ E. W. Plummer, J. W. Gadzuk, and R. D. Young, Solid State Commun. 7, 487 (1969).

17 J. W. Gadzuk and E. W. Plummer, Rev. Mod. Phys. 45, 487 (1973).

18 J. W. Gadzuk, Phys. Rev. B 47, 12832 (1993).

19 Single Charge Tunneling: Coulomb Blockade Phenomena in Nanostructures, edited by H. Grabert and M. H. Devoret (Plenum, New York, 1992).

${ }^{20}$ D. Goldhaber-Gordon, H. Shtrikman, D. Mahalu, D. Abusch-Magder, U. Meirav, and M. A. Kastner, Nature 391, 156 (1998).

${ }^{21}$ V. Madhavan, W. Chen, T. Jamneala, M. F. Crommie, and N. S. Wingreen, Science 280, 567 (1998).

22 J. Li, W. D. Schneider, R. Berndt, and B. Delley, Phys. Rev. Lett. 80, 2893 (1998).

23 J. Bardeen, Phys. Rev. Lett. 2, 57 (1961).

${ }^{24}$ J. Tersoff and D. R. Hamann, Phys. Rev. B 31, 805 (1985).

25 S. Gao, M. Persson, and B. I. Lundqvist, Phys. Rev. B 55, 4825 (1997).

${ }^{26}$ G. D. Mahan, Many-Particle Physics (Plenum, New York, 1990).

${ }^{27}$ G. F. Koster and J. C. Slater, Phys. Rev 96, 1208 (1954).

${ }^{28}$ P. W. Anderson, Phys. Rev. 124, 41 (1961).

${ }^{29}$ U. Fano, Phys. Rev. 124, 1866 (1961).

${ }^{30}$ C. Cohen-Tannoudji, J. Dupont-Roc, and G. Grynberg, Atom-Photon Interactions (John Wiley, New York, 1992).

${ }^{31}$ T. Kawasaka, H. Kasai, W. A. Dino, and A. Okiji, J. Appl. Phys 86, 6970 (1999).

32 A. Schiller and S. Hershfield, Phys. Rev. B 61, 9036 (2000).

${ }^{33}$ D. Penn, R. Gomer, and M. H. Cohen, Phys. Rev. B 5, 768 (1972).

${ }^{34}$ C. J. Chen, Phys. Rev. Lett. 65, 448 (1990).

${ }^{35}$ C. J. Chen, J. Vac. Sci. Technol. A 9, 44 (1991).

${ }^{36}$ N. D. Lang, A. Yacoby, and Y. Imry, Phys. Rev. Lett. 63, 1499 (1989).

${ }^{37}$ U. Wille, Phys. Rev. B 50, 1888 (1994).

${ }^{38}$ J. W. Gadzuk, Surf. Sci. 6, 133 (1967).

${ }^{39}$ P. Nordlander and J. C. Tully, Phys. Rev. B 42, 5564 (1990).

40 A. G. Borisov, R. Zimny, D. Teillet-Billy, and J. P. Gauyacq, Phys. Rev. A 53, 2457 (1996).

${ }^{41}$ P. Sautet, Surf. Sci. 374, 406 (1997).

42 M. Plihal, D. C. Langreth, and P. A. Nordlander, Phys. Rev. B 59, 13322 (1999).

${ }^{43}$ H. Eyring, J. Walter, and G. E. Kimball, Quantum Chem- istry (John Wiley, New York, 1944).

${ }^{44}$ I. N. Levine, Quantum Chemistry, 5th edition (Prentice Hall, New Jersey, 2000).

${ }^{45}$ L. V. Keldysh, J. Exptl. Theoret. Phys. (USSR) 47, 1515 (1964), [English Translation: Soviet Physics JETP 20, 1018 (1965)].

${ }^{46}$ L. P. Kadanoff and G. Baym, Quantum Statistical Mechanics (Benjamin, New York, 1962).

${ }^{47}$ D. C. Langreth, in Linear and Nonlinear Electron Transport in Solids, edited by J. T. Devreese and V. E. van Doren (Plenum, New York, 1976).

${ }^{48}$ H. Haug and A.-P.Jauho, Quantum Kinetics in Transport and Optics of Semiconductors (Springer, Berlin, Heidelberg, New York, 1996).

${ }^{49}$ M. Plihal and D. C. Langreth, Phys. Rev. B 58, 2191 (1998).

50 T. Kawasaka and H. K. A. Okiji, Phys. Lett. A 250, 403 (1998).

${ }^{51}$ N. S. Wingreen and Y. Meir, Phys. Rev. B 49, 11040 (1994).

52 N. Sivan and N. S. Wingreen, Phys. Rev. B 54, 11622 (1996).

${ }^{53}$ E. W. Plummer and W. Eberhardt, Adv. Chem. Phys. 49, 533 (1982).

${ }^{54}$ S. D. Kevan and R. H. Gaylord, Phys. Rev. B 36, 5809 (1987).

55 J. W. Gadzuk, J. Vac. Sci. Technol. 9, 591 (1972).

${ }^{56}$ S. D. Kevan and R. H. Gaylord, Phys. Rev. Lett. 57, 2975 (1986).

${ }^{57}$ M. P. Everson and R. C. Jaklevic, J. Vac. Sci. Technol 8, 3662 (1990).

${ }^{58}$ W. Chen, V. Madhavan, T. Jamneala, and M. F. Crommie, Phys. Rev. Lett 80, 1469 (1998).

${ }^{59}$ M. F. Crommie, C. P. Lutz, and D. M. Eigler, Science 262, 218 (1993).

${ }^{60}$ M. F. Crommie, C. P. Lutz, D. M. Eigler, and E. J. Heller, Physica D 83, 98 (1995).

${ }^{61}$ L. Bürgi, O. Jeandupeux, H. Brune, and K. Kern, Phys. Rev. Lett. 82, 4516 (1999).

${ }^{62}$ Y. Hasegawa and P. Avouris, Phys. Rev. Lett. 71, 1993 (1993).

63 P. Avouris, I.-W. Lyo, and P. Molinas-Mata, Chem. Phys. Lett. 240, 423 (1995).

${ }^{64}$ H. C. Manoharan, C. P. Lutz, and D. M. Eigler, Nature 403, 512 (2000).

${ }^{65}$ P. Coleman, Phys. Rev. B 29, 3035 (1984).

${ }^{66}$ N. D. Lang, Phys. Rev. Lett. 58, 45 (1987).

67 T. Jamneala, V. Madhavan, W. Chen, and M. F. Crommie, Phys. Rev. B 61, 9990 (2000).

${ }^{68}$ M. Plihal, D. C. Langreth, and P. A. Nordlander, Phys. Rev. B 61, 13341 (2000). 